\title{
Perturbation theory for nearly integrable multi-component nonlinear PDEs
}

\author{
V. S. Shchesnovich ${ }^{a)}$ \\ Department of Mathematics and Applied Mathematics, University of Cape \\ Town, Private Bag 7701 Rondebosch, South Africa ${ }^{b)}$ \\ (Received ) \\ The Riemann-Hilbert problem associated with the integrable PDE is used \\ as a nonlinear transformation of the nearly integrable PDE to the spectral \\ space. The temporal evolution of the spectral data is derived with account \\ for arbitrary perturbations and is given in the form of exact equations, \\ which generate the sequence of approximate ODEs in successive orders \\ with respect to the perturbation. For vector nearly integrable PDEs, \\ embracing the vector NLS and complex modified KdV equations, the main \\ result is formulated in a theorem. For a single vector soliton the evolution \\ equations for the soliton parameters and first-order radiation are given in \\ explicit form.
}

\footnotetext{
${ }^{a)}$ Electronic mail: valery@maths.uct.ac.za

${ }^{b)}$ On leave from the Division for Optical Problems in Information Technologies, National Academy of Sciences of Belarus, Zhodinskaya St. 1/2, 220141 Minsk, Belarus
} 


\section{INTRODUCTION}

Multi-component (or coupled) nonlinear PDEs had been a subject of considerable interest for many years (see, for instance, Ref. 1, and references therein). Recent revival of interest in multi-component PDEs is due to new discoveries and technological advances in nonlinear optics and physics of condensed matter. An important example is the incoherent spatial optical solitons, or self-trapped spatially incoherent light beams, recently experimentally observed in nonlinear media [2], which are described by the multi-component nonlinear Schrödinger (NLS) equations [3]. Another example of possible application of the coupled NLS equations is the creation and dynamics of solitary waves in the multispecies BoseEinstein condensates [4]. Similar models of coupled nonlinear PDEs appear in the wavelength division multiplexing, i.e., copropagation of pulses in an optical fibre on beams with different wavelengthes [5-7] and in other important applications $[8,9]$.

Some of the multi-component models are integrable. Integrable multi-component PDEs have another specific feature, which makes them important for applications as zeroapproximation models for analytical description of the real phenomena. It has been known for quite some time that dimensional reductions of matrix generalizations of the integrable PDEs, such as the NLS and KdV equations, can produce a variety of new integrable equations [10]. For instance, some of the coupled NLS equations are integrable reductions of the general matrix NLS equation. The $N$-dimensional matrix NLS equation is the simplest integrable PDE associated with the $(N+1)$-dimensional Zakharov-Shabat spectral problem [11-13]. Recently a variety of integrable coupled higher-order NLS equations was discovered [14-16], which are important in view of applications to the soliton propagation of sub-picosecond pulses in optical fibre [17-19]. Some of these integrable PDEs arise as dimensional reductions of the matrix complex modified $\mathrm{KdV}(\mathrm{cmKdV})$ equation, which is also associated with the Zakharov-Shabat spectral problem.

In most cases, the multi-component $\mathrm{PDE}$ is not integrable. However, frequently the terms destroying integrability contain small parameters and the non-integrable equation can 
be considered as a perturbation of the integrable one. In this case, a perturbation theory is required for analytical description of the effect of small perturbations. For instance, one is especially interested in the dynamics of solitons in nearly integrable PDEs. Soliton solutions to multi-component equations have many parameters, and their evolution may exhibit a variety of new interesting regimes. Therefore, it is necessary to have at hand a perturbation theory for multi-component nearly integrable equations. Such perturbation theory is developed in the present paper.

Perturbation theory for nearly integrable PDEs has a long history [20-45]. There are two basic approaches in the perturbation theory based on the IST method. The first one originated in works of Kaup [20] and Karpman and Maslov [21], where the perturbation theory was developed for nearly integrable PDEs associated in the integrable limit with the $2 \times 2$ matrix Zakharov-Shabat spectral problem. Quite different approach originated from works [22-26]. It was found that an integro-differential operator, generating the whole hierarchy of integrable PDEs related to a given spectral problem, called the recursion operator, has a complete set of eigenfunctions, which can be used for the perturbation expansion. Several other methods, not related to the IST, were applied for description of the perturbed soliton dynamics. For instance, a method based on the Green functions was developed in Ref. 29. The IST-independent perturbation theories for solitons are usually referred to as the direct perturbation theories (see for instance, Ref. 40 and references therein). However, notwithstanding the long history of the perturbation theory, with rare exceptions, only the $2 \times 2$ matrix spectral problems were considered. It was noted that construction of the perturbation theory for higher-dimensional matrix spectral problems along the standard approach becomes technically more involved.

To overcome technical difficulties of the standard approach when dealing with multicomponent PDEs, the method based on the Riemann-Hilbert (RH) problem was proposed in Ref. 41, where the perturbation theory was developed for the Zakharov-Shabat spectral problem of an arbitrary matrix dimension. The $\mathrm{RH}$ problem was used before for construction of the perturbation theory for the Landau-Lifschitz equation [30], the NLS and Maxwell- 
Bloch equations [34], which are integrable by the $2 \times 2$ matrix spectral problem. The approach of Ref. 41 was applied to the Manakov system [42] (i.e., the two-component NLS equation), modified NLS equation [43] and massive Thirring model [44]. These examples demonstrate that the perturbation theory based on the RH problem always works. Recently, the $\mathrm{RH}$ problem was applied to nearly integrable equations on the half line, arising from the singular dispersion relations [45]. In Refs. 41-45 the perturbation-induced evolution equations for the spectral data were derived with the help of some matrix functional (below, the evolution functional $\Pi(x, t, k))$. It is important to emphasize that the form of the evolution functional is invariant under the gauge transformations of the considered PDE [43]. Thus, once constructed, the evolution functional is valid not only for the whole hierarchy of PDEs associated with a given spectral problem, but also for their images under the gauge transformations. Writing the dispersion law, generating the spectral problem, in an abstract form $\Lambda(k)$ (see the next section for details) we discover that the form of the evolution functional remains invariant under the change of the spectral problem as well. This invariance trivially extends to the general initial-boundary value problems. For instance, for the halfline, where one would expect a difference, we have found that the evolution functional has similar form [45]. Therefore, it seems that the approach based on the evolution functional is universal for construction of the perturbation theories for nearly integrable PDEs. It is also technically simple. Derivation of the perturbation-induced evolution equations for the spectral data using the evolution functional reduces to calculation of integrals.

This paper is a further development of Ref. 41. The previous results are substantially advanced. In particular, the evolution equations for the spectral data are considerably simplified with the help of some identities found for the evolution functional. We start section $[1]$ with a brief discussion of the multi-component integrable PDEs associated with the Zakharov-Shabat spectral problem. We consider two examples, the matrix NLS and cmKdV equations, however, our approach is valid for many other multi-component PDEs. We have not made an attempt to give a complete exposition of the properties of integrable equations. We need only the Lax representation. Hence a way of deriving the Lax pair for 
an integrable PDE from the dispersion relation of its linearization is briefly indicated. For completeness of the exposition, a detailed derivation of the $\mathrm{RH}$ problem is given in section III. Solution of the RH problem for multi-component equations involves some technicalities, which are discussed and detailed derivations are provided in the Appendices. We derive evolution equations for the spectral data with account for perturbations in section $\amalg \nabla$. There, for an important special case of the vector nearly integrable PDEs the main result of this paper is formulated in a theorem. In the final section $\square$ the equations of the first-order perturbation theory for a single vector soliton are given in explicit form.

\section{PRELIMINARIES: INTEGRABLE MULTI-COMPONENT NONLINEAR PDES}

Here we briefly discuss integrable PDEs with emphasis on the multi-component equations whose reductions are important for applications. In particular, we consider the matrix nonlinear Schrödinger and complex modified Korteweg-de Vries equations. We do not try to review this subject, for general considerations the reader can consult, for instance, Refs. 1, 10-13, 46-53 and the references therein. The purpose of this section is to remind some of the basic notions in the IST method. Though the approach below can be applied to any nonlinear PDE solvable by the RH problem, we restrict the consideration to the integrable equations associated with the $N$-dimensional Zakharov-Shabat spectral problem (1).

Consider the integrable PDEs which arise as the compatibility condition for the following $N \times N$ matrix linear system (Lax pair)

$$
\begin{gathered}
\partial_{x} \Phi=i k[A, \Phi]+i Q(x, t) \Phi \equiv \Phi \Lambda(k)+U(x, t, k) \Phi, \\
\partial_{t} \Phi=i \omega(k) \Phi A+V(x, t, k) \Phi \equiv \Phi \Omega(k)+V(x, t, k) \Phi,
\end{gathered}
$$

with

$$
A=\left(\begin{array}{cc}
I_{n} & 0 \\
0 & -I_{N-n}
\end{array}\right), \quad Q=\left(\begin{array}{cc}
0 & \mathbf{q} \\
\overline{\mathbf{q}} & 0
\end{array}\right),
$$




$$
\mathbf{q}=\left(\begin{array}{cccc}
q_{11} & q_{12} & \ldots & q_{1, N-n} \\
q_{21} & q_{22} & \ldots & q_{2, N-n} \\
\vdots & \vdots & & \vdots \\
q_{n 1} & q_{n 2} & \ldots & q_{n, N-n}
\end{array}\right), \quad \overline{\mathbf{q}}=\left(\begin{array}{cccc}
\bar{q}_{11} & \bar{q}_{21} & \ldots & \bar{q}_{n 1} \\
\bar{q}_{12} & \bar{q}_{22} & \ldots & \bar{q}_{n 2} \\
\vdots & \vdots & & \vdots \\
\bar{q}_{1, N-n} & \bar{q}_{2 n} & \ldots & \bar{q}_{n, N-n}
\end{array}\right)
$$

where $\Lambda(k)=-i k A$ and $\Omega=i \omega(k) A$ are the dispersion laws, $Q$ is called the potential. Here the overline does not mean complex conjugation by default, e.g., in general, the functions $q_{i j}$ and $\bar{q}_{i j}$ are not considered as complex conjugate to each other. When the overline does denote complex conjugation in the text below, each time it will be specially indicated. This special case corresponds to the Hermitian potential $Q, Q^{\dagger}=Q$ or $\mathbf{q}^{\dagger}=\overline{\mathbf{q}}$, and it will be referred to as the involution. The temporal evolution equation (2) is specified by choice of the dispersion relation $\omega(k)$ in the following manner. For simplicity, let the dispersion relation be polynomial $\omega(k)=\sum_{p=1}^{M} w_{p} k^{p}$, then

$$
V(k)=-\mathcal{P}\left\{\Phi \Omega \Phi^{-1}\right\} \equiv-\Omega(k)+\sum_{p=0}^{M-1} V_{p} k^{p} .
$$

Here the matrix function $\Phi(k)$ is expanded into the asymptotic series:

$$
\Phi(k)=I+k^{-1} \Phi^{(1)}+k^{-2} \Phi^{(2)}+\ldots, \quad k \rightarrow \infty,
$$

and the operator $\mathcal{P}$ takes the polynomial in $k$ part of $\Phi \Omega \Phi^{-1}$ on the asymptotics. For example, the Zakharov-Shabat spectral problem (1) is derived in this way

$$
U=-\mathcal{P}\left\{\Phi \Lambda \Phi^{-1}\right\}=i k A+i\left[\Phi^{(1)}, A\right]
$$

with the obvious relation

$$
Q=\left[\Phi^{(1)}, A\right]
$$

Hence, the Lax pair satisfies the property $\operatorname{Tr} U=-\operatorname{Tr} \Lambda$ and $\operatorname{Tr} V=-\operatorname{Tr} \Omega$.

The integrable nonlinear PDE related to the Lax pair (11)-(2) is given by the compatibility condition (in our case, polynomial in $k$ )

$$
i \partial_{t} Q-\partial_{x} V+[i k A+i Q, V]=0
$$


via setting $k=0$, while the positive powers of $k$ supply the expressions of the coefficients $V_{p}$ in (3) as functions of the potential $Q$ and its $x$-derivatives, $V_{p}=V_{p}\left(Q, Q_{x}, Q_{x x}, \ldots\right)$. For instance, choosing $\omega(k)=2 k^{2}$ we obtain the well-known matrix nonlinear Schrödinger equation. Indeed, in this case

$$
V=-2 i k^{2} A-2 i k Q-A Q_{x}+i A Q^{2}
$$

and the equation (5) becomes

$$
i A Q_{t}+Q_{x x}+2 Q^{3}=0 .
$$

For a complete classification of the matrix integrable NLS equations with various reductions to Hermitian symmetric spaces consult Ref. 10. A particular important case of equation (6) is the vector NLS equation, a generalization of the two-component vector NLS, which was shown to be integrable by Manakov [12]. The vector NLS equation corresponds to the Hermitian potential and the reduction $n=N-1$ (see the expression for $A$ ). In this case we have

$$
Q=\left(\begin{array}{cccc}
0 & \ldots & 0 & q_{1} \\
\vdots & & \vdots & \vdots \\
0 & \ldots & 0 & q_{n} \\
\bar{q}_{1} & \ldots & \bar{q}_{n} & 0
\end{array}\right)
$$

and matrix equation (6) becomes the vector NLS equation:

$$
i \partial_{t} q_{l}+\partial_{x}^{2} q_{l}+2\left(\sum_{j=1}^{n}\left|q_{j}\right|^{2}\right) q_{l}=0, \quad l=1, \ldots, n .
$$

Let us consider another important example of multi-component nonlinear integrable equations. It is given by setting $\omega(k)=4 k^{3}$. After simple computation we get

$$
V=-4 i k^{3} A+4 i k^{2} Q+2 i k A\left(i Q_{x}+Q^{2}\right)-i Q_{x x}+\left[Q, Q_{x}\right]-2 i Q^{3}
$$

which produces the matrix cmKdV equation

$$
Q_{t}+Q_{x x x}+3\left(Q^{2} Q_{x}+Q_{x} Q^{2}\right)=0 .
$$


Combining together the considered two dispersion laws, i.e., letting $\omega(k)=4 i \epsilon k^{3}+2 i \beta k^{2}$, one can obtain

$$
i Q_{t}+\beta A\left(Q_{x x}+2 Q^{3}\right)+i \epsilon Q_{x x x}+3 i \epsilon\left(Q^{2} Q_{x}+Q_{x} Q^{2}\right)=0
$$

a special case of the (generally, non-integrable) matrix higher-order NLS equation

$$
i E_{z}+A\left(\alpha_{1} E_{\tau \tau}+\alpha_{2} E^{3}\right)+i\left\{\alpha_{3} E_{\tau \tau \tau}+\alpha_{4}\left(E^{3}\right)_{\tau}+\alpha_{5}\left(E^{2}\right)_{\tau} E\right\}=0,
$$

Our examples are just illustrative. There is many other integrable matrix PDEs which we do not mention here. However, the perturbation theory developed in section IV applies to such PDEs also.

\section{RIEMANN-HILBERT PROBLEM FOR MULTI-COMPONENT PDES}

In this section we derive the $\mathrm{RH}$ problem for the multi-component integrable PDEs and discuss its solution (for more details consult Refs. 46, 54-57). We are interested in the initialvalue problem for nonlinear PDEs on the whole real line with the asymptotically vanishing conditions (Cauchy problem) $q_{i j} \rightarrow 0$ as $|x| \rightarrow \infty$. The vanishing asymptotics allows us to concentrate entirely on the spectral equation (1) in derivation of the $\mathrm{RH}$ problem, while $t$ dependence enters parametrically in our approach (for the $\mathrm{RH}$ problem for initial-boundary value problems consult Ref. 47). Below we omit the explicit $t$-dependence for simplicity of the presentation. To begin with, let us summarize the properties of the spectral problem (1). Define the following $N \times N$ matrix projectors

$$
H_{1}=\operatorname{diag}\left(I_{n}, 0\right), \quad H_{2}=\operatorname{diag}\left(0, I_{N-n}\right) .
$$

Then $A=H_{1}-H_{2}$ and any matrix can be decomposed into the sum of two matrices, commuting and anti-commuting with $A$ :

$$
\Phi=\Phi^{(c)}+\Phi^{(a)}, \quad \Phi^{(c)}=H_{1} \Phi H_{1}+H_{2} \Phi H_{2}, \quad \Phi^{(a)}=H_{1} \Phi H_{2}+H_{2} \Phi H_{1},
$$


where $\left[A, \Phi^{(c)}\right]=0$ and $\left\{A, \Phi^{(a)}\right\}=0$. We will use the block-index notations for the decomposition of matrix $\Phi$ with respect to the projectors $H_{1}$ and $H_{2}$ :

$$
\Phi=\left(\begin{array}{cc}
\Phi_{I, I} & \Phi_{I, I I} \\
\Phi_{I I, I} & \Phi_{I I, I I}
\end{array}\right) .
$$

The $\mathrm{RH}$ problem is a one-to-one mapping (nonlinear Fourier transform) between the set of smooth (e.g., belonging to the Schwartz space) potentials $Q(x)$ and some set of the spectral data. To identify the RH problem one must construct two solutions, one, $\Phi_{+}(x, k)$, to the spectral equation (11) and the other, $\Phi_{-}^{-1}(x, k)$, to the adjoint equation (the second function is inverse of some matrix function satisfying (1), hence the "-1" in its definition), holomorphic with respect to the spectral parameter $k$ in some complementary domains covering the whole complex $k$-plane. Such solutions can be built from the columns and rows of the Jost solutions $J_{ \pm}$, i.e., solutions defined by the asymptotic conditions: $J_{ \pm}(x, k) \rightarrow I$ as $x \rightarrow \pm \infty$.

As $\operatorname{Tr} Q=0$, letting $x \rightarrow \pm \infty$ we conclude that $\operatorname{det} J_{ \pm}=1$. Hence, the columns of either of the two Jost solutions give a linear basis in the space of solutions of the spectral equation. The inverse matrices $J_{ \pm}^{-1}$ satisfy the adjoint spectral equation:

$$
\partial_{x} \widetilde{\Phi}=i k[A, \widetilde{\Phi}]-i \widetilde{\Phi} Q
$$

Each column of $J_{ \pm}(x, k)$ and, respectively, row of $J_{ \pm}^{-1}(x, k)$ is holomorphic and bounded in either upper $(\operatorname{Im} k \geq 0)$ or lower $(\operatorname{Im} k \leq 0)$ half of the complex $k$-plane. Indeed, this can be easily seen from the Volterra integral equations for the Jost matrices written for the two blocks of columns:

$$
\begin{aligned}
& J_{ \pm}(x, k) H_{1}=H_{1}+i \int_{ \pm \infty}^{x} \mathrm{~d} \xi e^{-2 i k(x-\xi) H_{2}} Q(\xi) J_{ \pm}(\xi, k) H_{1} \\
& J_{ \pm}(x, k) H_{2}=H_{2}+i \int_{ \pm \infty}^{x} \mathrm{~d} \xi e^{2 i k(x-\xi) H_{1}} Q(\xi) J_{ \pm}(\xi, k) H_{2} .
\end{aligned}
$$


The columns of $J_{+}(k) H_{1}$ and $J_{-}(k) H_{2}$ are holomorphic and bounded in the upper half of the complex plane, while columns of $J_{+}(k) H_{2}$ and $J_{-}(k) H_{1}$ have the same property in the lower half plane. Similarly, the rows of $J_{ \pm}^{-1}$ satisfy integral equations

$$
\begin{aligned}
& H_{1} J_{ \pm}^{-1}(x, k)=H_{1}-i \int_{ \pm \infty}^{x} \mathrm{~d} \xi H_{1} J_{ \pm}^{-1}(\xi, k) Q(\xi) e^{2 i k(x-\xi) H_{2}} \\
& H_{2} J_{ \pm}^{-1}(x, k)=H_{2}-i \int_{ \pm \infty}^{x} \mathrm{~d} \xi H_{2} J_{ \pm}^{-1}(\xi, k) Q(\xi) e^{-2 i k(x-\xi) H_{1}}
\end{aligned}
$$

from which we immediately conclude that $H_{1} J_{+}^{-1}(k)$ and $H_{2} J_{-}^{-1}(k)$ are holomorphic and bounded in the upper half plane, while $H_{2} J_{+}^{-1}(k)$ and $H_{1} J_{-}^{-1}(k)$ have the same properties in the lower half plane.

On the real line, the Jost solutions are transformed into each other by the scattering matrix $S(k)$,

$$
J_{-}(x, k)=J_{+}(x, k) e^{i k x A} S(k) e^{-i k x A} .
$$

For the Hermitian potential, $Q^{\dagger}=Q$, the matrix Jost solutions satisfy the involution (here the overline means complex conjugation)

$$
J_{ \pm}^{\dagger}(x, k)=J_{ \pm}^{-1}(x, \bar{k})
$$

where the spectral parameter takes complex values in the upper or lower half plane depending on the considered column of the Jost matrix. In this case, the scattering matrix also satisfies the involution

$$
S^{\dagger}(k)=S^{-1}(k), \quad k \in \text { Re } .
$$

The holomorphic matrix functions $\Phi_{+}(k)$ and $\Phi_{-}^{-1}(k)$, satisfying equation (11) and (13), respectively, are given in terms of columns and rows of the Jost solutions:

$$
\Phi_{+}=J_{+} H_{1}+J_{-} H_{2}, \quad \Phi_{-}^{-1}=H_{1} J_{+}^{-1}+H_{2} J_{-}^{-1} .
$$


The above defined matrix functions are holomorphic and bounded in the upper and lower half planes, respectively. They have the following asymptotics

$$
\Phi_{ \pm}(x, k) \rightarrow I, \quad k \rightarrow \infty
$$

which follow from the Volterra integral equations for the Jost solutions. For the involution (15) the matrices $\Phi_{+}(k)$ and $\Phi_{-}^{-1}(k)$ are related via

$$
\Phi_{+}^{\dagger}(k)=\Phi_{-}^{-1}(\bar{k})
$$

These matrices can be conveniently expressed in terms of only one Jost solution and elements of the scattering matrix $S(k)$. Indeed,

$$
\begin{gathered}
\Phi_{+}=J_{+} e^{i k x A}\left(H_{1}+S H_{2}\right) e^{-i k x A} \equiv J_{-} e^{i k x A}\left(H_{2}+S^{-1} H_{1}\right) e^{-i k x A}, \\
\Phi_{-}^{-1}=e^{i k x A}\left(H_{1}+H_{2} S^{-1}\right) e^{-i k x A} J_{+}^{-1} \equiv e^{i k x A}\left(H_{2}+H_{1} S\right) e^{-i k x A} J_{-}^{-1} .
\end{gathered}
$$

Denote $S_{+}=H_{1}+S H_{2}, S_{-}=H_{2}+S^{-1} H_{1}$. These matrices provide a factorization of the scattering matrix: $S_{+}=S S_{-}$. Similarly, $\bar{S}_{+}=H_{1}+H_{2} S^{-1}$ and $\bar{S}_{-}=H_{2}+H_{1} S$, which also factorize the scattering matrix: $\bar{S}_{+} S=\bar{S}_{-}$. Then

$$
\begin{gathered}
\Phi_{+}=J_{+} e^{i k x A} S_{+} e^{-i k x A} \equiv J_{-} e^{i k x A} S_{-} e^{-i k x A}, \\
\Phi_{-}^{-1}=e^{i k x A} \bar{S}_{+} e^{-i k x A} J_{+}^{-1} \equiv e^{i k x A} \bar{S}_{-} e^{-i k x A} J_{-}^{-1} .
\end{gathered}
$$

The factorization matrices have the block-triangular structure. For instance, $S_{+}$and $\bar{S}_{+}$ are upper and lower block-triangular, respectively:

$$
S_{+}=\left(\begin{array}{cc}
I_{n} & \mathbf{b} \\
0 & \mathbf{a}
\end{array}\right), \quad \bar{S}_{+}=\left(\begin{array}{cc}
I_{n} & 0 \\
\overline{\mathbf{b}} & \overline{\mathbf{a}}
\end{array}\right),
$$

where $\mathbf{b}=S_{I, I I}, \mathbf{a}=S_{I I, I I}, \overline{\mathbf{b}}=\left(S^{-1}\right)_{I I, I}$, and $\overline{\mathbf{a}}=\left(S^{-1}\right)_{I I, I I}$. The following identity follows from these definitions 


$$
\overline{\mathbf{b}} \mathbf{b}+\overline{\mathbf{a}} \mathbf{a}=I_{N-n}, \quad k \in \text { Re. }
$$

For the involution (19) the factorizations satisfy

$$
S_{ \pm}^{\dagger}(k)=\bar{S}_{ \pm}(k), \quad k \in \operatorname{Re}
$$

Hence, $\overline{\mathbf{b}}=\mathbf{b}^{\dagger}$ and $\overline{\mathbf{a}}=\mathbf{a}^{\dagger}$ in the case of involution.

Considering the product $\Phi_{-}^{-1} \Phi_{+}$we obtain the problem of analytic factorization of a matrix $G(k)$ given on the real line, i.e., the matrix $\mathrm{RH}$ problem:

$$
\Phi_{-}^{-1}(x, k) \Phi_{+}(x, k)=e^{i k x A} G(k) e^{-i k x A}, \quad k \in \operatorname{Re}
$$

and $\Phi_{ \pm}(x, k) \rightarrow I$ for $k \rightarrow \infty$. Here the matrix $G=\bar{S}_{+} S_{+} \equiv \bar{S}_{-} S_{-}$reads

$$
G=\left(\begin{array}{cc}
I_{n} & \mathbf{b} \\
\overline{\mathbf{b}} & I_{N-n}
\end{array}\right) .
$$

As it was mentioned, the $\mathrm{RH}$ problem is a nonlinear mapping between the potential $Q(x)$ and the set of the spectral data, which are necessary for unique identification of the solution to (23). For instance, given a potential, one can obtain the spectral data by solving the spectral equation and its adjoint for $\Phi_{+}(x, k)$ and $\Phi_{-}^{-1}(x, k)$. Conversely, by asymptotic expansion of $\Phi_{ \pm}(x, k)$ as $k \rightarrow \infty$ one recovers the potential. The asymptotic expansion of $\Phi_{ \pm}(x, k)$ can be derived via integration by parts (in the blocks with $\left.e^{ \pm 2 i k x}\right)$ in the Volterra integral equations for $J_{ \pm}$and $J_{ \pm}^{-1}$. It reads

$$
\Phi_{ \pm}(x, k)=I-\left(A Q(x)+i \int_{-\infty}^{x} \mathrm{~d} \xi Q^{2}(\xi) H_{2}+i \int_{x}^{\infty} \mathrm{d} \xi Q^{2}(\xi) H_{1}\right) \frac{1}{2 k}+\mathcal{O}\left(\frac{1}{k^{2}}\right) .
$$

Hence, we obtain (cf. with (四)

$$
Q(x)=\lim _{k \rightarrow \infty} k\left[\Phi_{+}(x, k), A\right]=\lim _{k \rightarrow \infty} k\left[A, \Phi_{-}^{-1}(x, k)\right] .
$$




\section{Solution of the RH problem: Normalization}

Let us discuss the way of solution of the RH problem. The coordinate dependence is not important for this purpose and it is omitted below. In general, the determinants det $\Phi_{+}(k)$ and $\operatorname{det} \Phi_{-}^{-1}(k)$ have zeros and the $\mathrm{RH}$ problem is said to be non-regular or with zeros. Note that the determinants do not depend on $x$ as it readily seen from equations (1) and (13). We consider only the RH problem with zero index, i.e.,

$$
\int_{-\infty}^{\infty} \mathrm{d} \ln \{\operatorname{det} G(k)\}=0
$$

assuming that $G(k)$ is non-degenerate. Since $\Phi_{ \pm} \rightarrow I$ as $k \rightarrow \infty$, the index is equal to the difference between the number of zeros in the upper and lower half planes. Let $k_{1}, \ldots, k_{M}$ and $\bar{k}_{1}, \ldots, \bar{k}_{M}$ be zeros (where some may be equal) of $\operatorname{det} \Phi_{+}(k)$ and $\operatorname{det} \Phi_{-}^{-1}(k)$, respectively. Two conditions are imposed on the zeros. First, the geometric multiplicity of a zero must be equal to its order (which we will refer to as the algebraic multiplicity). Here the geometric multiplicity of $k_{j}$ is defined as the dimension $d_{j}$ of the null space of $\Phi_{+}\left(k_{j}\right)$, i.e., $d_{j}=N-\operatorname{rank} \Phi_{+}\left(k_{j}\right)$. Trivially, the two multiplicities are equal to 1 in the case of simple zeros. In the case of involution zeros of the $\mathrm{RH}$ problem come in complex conjugate pairs (due to formula (19)), with coinciding algebraic (and geometric) multiplicities within each pair. (Due to the involution the index is equal to zero, in this case $\operatorname{det} G(k)$ is real and definite.) Second, as we are mainly interested in the Hermitian potentials $Q$, i.e., in the case of the involution, we will consider only paired zeros $k_{j}$ and $\bar{k}_{j}, j=1, \ldots, s \leq M$, whose algebraic multiplicities are equal, however without assuming them to be complex conjugate to each other. The algebraic multiplicity $\nu_{j}$ of $k_{j}$ always satisfies the following inequality (see Appendix A)

$$
\nu_{j} \geq N-\operatorname{rank} \Phi_{+}\left(k_{j}\right)=d_{j}
$$

while $\operatorname{rank} \Phi_{+} \geq \max (n, N-n)$ by construction of $\Phi_{+}$(17). For instance, if $n=N-1$, there

can be not more than one vector in the null space of $\Phi_{+}(k)$, i.e., $d_{j}=1$ for all $j=1, \ldots, s$. 
Hence, for this reduction, zeros of $\operatorname{det} \Phi_{+}(k)$ must be simple to satisfy the equal multiplicity condition. Similar for zeros of $\Phi_{-}^{-1}(k)$. (More detailed consideration of the multiplicities of zeros is placed in Appendix A.)

Let $\operatorname{det} \Phi_{+}(k)$ and $\operatorname{det} \Phi_{-}^{-1}(k)$ have zeros $k_{1}, \ldots, k_{s}$ and $\bar{k}_{1}, \ldots \bar{k}_{s}$, respectively, with the (algebraic and geometric) multiplicities $\nu_{1}, \ldots, \nu_{s}$. Exactly $\nu_{j}$ independent vector-columns $\left|p_{l}^{(j)}\right\rangle$ (vector-rows $\left\langle\bar{p}_{l}^{(j)}\right|$ ), where $l=1, \ldots, \nu_{j}$, correspond to each zero $k_{j}$ (respectively, $\bar{k}_{j}$ ), such that

$$
\Phi_{+}\left(k_{j}\right)\left|p_{l}^{(j)}\right\rangle=0, \quad\left\langle\bar{p}_{l}^{(j)}\right| \Phi_{-}^{-1}\left(\bar{k}_{j}\right)=0 .
$$

To specify a unique solution to the RH problem, additionally to the continuous datum $G(k)$, the set of the discrete data $\left\{k_{j},\left|p_{l}^{(j)}\right\rangle, \bar{k}_{j},\left\langle\bar{p}_{l}^{(j)}\right|, l=1, \ldots, \nu_{j}, j=1, \ldots, s\right\}$ must be given. This becomes evident from the fact that the $\mathrm{RH}$ problem with zeros (i.e., non-regular) can be regularized, i.e., reduced to a modified $\mathrm{RH}$ problem without zeros by factoring them out with some rational matrix $\Gamma(k)$ :

$$
\Phi_{+}(k)=\phi_{+}(k) \Gamma(k), \quad \Phi_{-}^{-1}(k)=\Gamma^{-1}(k) \phi_{-}^{-1}(k) .
$$

The regularized $\mathrm{RH}$ problem reads

$$
\phi_{-}^{-1}(k) \phi_{+}(k)=\Gamma(k) e^{i k x A} G(k) e^{-i k x A} \Gamma^{-1}(k), \quad k \in \operatorname{Re}
$$

and $\phi_{ \pm}(k) \rightarrow I$ as $k \rightarrow \infty$.

The rational matrix functions $\Gamma(k)$ and $\Gamma^{-1}(k)$, (for details see Appendix B)

$$
\Gamma=I-\sum_{j, l ; i, m} \frac{\left|p_{m}^{(i)}\right\rangle\left(D^{-1}\right)_{i m, j l}\left\langle\bar{p}_{l}^{(j)}\right|}{k-\bar{k}_{j}}, \quad \Gamma^{-1}=I+\sum_{j, l ; i, m} \frac{\left|p_{l}^{(j)}\right\rangle\left(D^{-1}\right)_{j l, i m}\left\langle\bar{p}_{m}^{(i)}\right|}{k-k_{j}}
$$

where

$$
D_{i m, j l}=\frac{\left\langle\bar{p}_{m}^{(i)} \mid p_{l}^{(j)}\right\rangle}{k_{j}-\bar{k}_{i}}
$$

have the same zeros as $\Phi_{+}(k)$ and $\Phi_{-}^{-1}(k)$, respectively, and the same null spaces:

$$
\Gamma\left(k_{j}\right)\left|p_{l}^{(j)}\right\rangle=0, \quad\left\langle\bar{p}_{l}^{(j)}\right| \Gamma^{-1}\left(\bar{k}_{j}\right)=0, \quad l=1, \ldots, \nu_{j}, \quad j=1, \ldots, s .
$$


Moreover, $\operatorname{det} \Gamma=\prod_{j=1}^{s}\left(\frac{k-k_{j}}{k-\bar{k}_{j}}\right)^{\nu_{j}}$. Precisely these properties allow us to factor out zeros of $\Phi_{+}(x, k)$ and $\Phi_{-}^{-1}(x, k)$.

It is important to emphasize that there is a freedom in choice of the basis vectors spaning the null spaces. Indeed, it is easy to verify that the regularization matrix $\Gamma$ is invariant under the transformations:

$$
\left|p_{l}^{(j)}\right\rangle \rightarrow \sum_{m=1}^{\nu_{j}}\left|p_{m}^{(j)}\right\rangle M_{m l}^{(j)}, \quad\left\langle\bar{p}_{l}^{(j)}\right| \rightarrow \sum_{m=1}^{\nu_{j}} \bar{M}_{l m}^{(j)}\left\langle\bar{p}_{m}^{(j)}\right|
$$

where $M^{(j)}$ and $\bar{M}^{(j)}$ are arbitrary non-degenerate $\nu_{j} \times \nu_{j}$-matrices. For the involution, due to equation (19) we can choose $\left\langle\bar{p}_{l}^{(j)}|=| p_{l}^{(j)}\right\rangle^{\dagger}$. This evidently leads to

$$
\Gamma^{\dagger}(k)=\Gamma^{-1}(\bar{k})
$$

The $(x, t)$-dependence of the RH data can be found in the following way. The $t$ dependence of the continuous datum (which does not depend on $x$ ) can be derived from equations (23) and (21). We obtain

$$
\partial_{t} G=[G, \Omega]
$$

This equation can be cast in a more explicit form:

$$
\partial_{t} \mathbf{b}=-2 i \omega(k) \mathbf{b}, \quad \partial_{t} \overline{\mathbf{b}}=2 i \omega(k) \overline{\mathbf{b}}
$$

Recalling that $\operatorname{Tr} Q=0$ and $\operatorname{Tr} V=-\operatorname{Tr} \Omega$, one can verify that the determinants of $\Phi_{ \pm}$do not depend on the co-ordinates. Therefore, the zeros are co-ordinate independent as well:

$$
\partial_{x} k_{j}=\partial_{t} k_{j}=0, \quad \partial_{x} \bar{k}_{j}=\partial_{t} \bar{k}_{j}=0, \quad j=1, \ldots, s
$$

Now, let us derive the co-ordinate dependence of the vector-parameters. Differentiating equation (26) we obtain

$$
\Phi_{+}\left(k_{j}\right)\left(\partial_{x}\left|p_{l}^{(j)}\right\rangle-i k_{j} A\left|p_{l}^{(j)}\right\rangle\right)=0, \quad \Phi_{+}\left(k_{j}\right)\left(\partial_{t}\left|p_{l}^{(j)}\right\rangle+\Omega\left(k_{j}\right)\left|p_{l}^{(j)}\right\rangle\right)=0
$$

Therefore 


$$
\partial_{x}\left|p_{l}^{(j)}\right\rangle=i k_{j} A\left|p_{l}^{(j)}\right\rangle+\sum_{m=1}^{\nu_{j}} F_{l m}^{(j)}\left|p_{m}^{(j)}\right\rangle, \quad \partial_{t}\left|p_{l}^{(j)}\right\rangle=-\Omega\left(k_{j}\right)\left|p_{l}^{(j)}\right\rangle+\sum_{m=1}^{\nu_{j}} K_{l m}^{(j)}\left|p_{m}^{(j)}\right\rangle,
$$

where $F$ and $K$ are some matrices. Using a suitable invariance transformation of the type (30) one can put $F=K=0$ without loss of generality. Hence we have

$$
\partial_{x}\left|p_{l}^{(j)}\right\rangle=i k_{j} A\left|p_{l}^{(j)}\right\rangle, \quad \partial_{t}\left|p_{l}^{(j)}\right\rangle=-\Omega\left(k_{j}\right)\left|p_{l}^{(j)}\right\rangle
$$

Similarly

$$
\partial_{x}\left\langle\bar{p}_{l}^{(j)}\right|=-\left\langle\bar{p}_{l}^{(j)}\right| i \bar{k}_{j} A, \quad \partial_{t}\left\langle\bar{p}_{l}^{(j)}\right|=\left\langle\bar{p}_{l}^{(j)}\right| \Omega\left(\bar{k}_{j}\right) .
$$

Some comments are needed on the reconstruction of the potential $Q$. First of all, the soliton part of the potential is given by the rational matrix function $\Gamma(k)$ (29). The pure soliton potentials are also called reflectionless, they solve the simplest $\mathrm{RH}$ problem with $G=I$, i.e., with zero reflection coefficients: $\mathbf{b}(k)=\overline{\mathbf{b}}(k)=0$. The discrete RH data have the following meaning. Zeros provide the amplitudes and velocities of the solitons, while the null vectors give their initial position, polarization and phase parameters. Second, the radiation part of the potential is given by the solution to the regularized $\mathrm{RH}$ problem (28). The continuous $\mathrm{RH}$ data $\mathbf{b}(k)$ and $\overline{\mathbf{b}}(k)$ represent the nonlinear spectral densities of radiation. The regular $\mathrm{RH}$ problem is equivalent to some matrix integral equation of the Fredholm type (for instance, consult Ref. 46). Though the solution cannot be given in explicit form, its properties can be explored by the standard technique of the theory of Fredholm integral equations.

For the following, it is convenient to introduce $x$-independent null vectors $\left|P_{l}^{(j)}\right\rangle$ and $\left\langle\bar{P}_{l}^{(j)}\right|$ setting

$$
\left|p_{l}^{(j)}\right\rangle=e^{i k_{j} x A}\left|P_{l}^{(j)}\right\rangle, \quad\left\langle\bar{p}_{l}^{(j)}\right|=\left\langle\bar{P}_{l}^{(j)}\right| e^{-i \bar{k}_{j} x A} .
$$

Then, we have an $x$-independent set of the RH data, which we will call the spectral data: $\left\{\mathbf{b}(k), \overline{\mathbf{b}}(k), k_{j},\left|P_{l}^{(j)}\right\rangle, \bar{k}_{j},\left\langle\bar{P}_{l}^{(j)}\right|, l=1, \ldots, \nu_{j}, j=1, \ldots, s\right\}$.

As the illustrative example, consider one-soliton solution. It is given by $\Gamma(k)$ having only one pole, say $k_{1}=i \eta+\xi$. In this case 


$$
Q=\left(k_{1}-\bar{k}_{1}\right)\left[A, P_{r}\right], \quad P_{r}=\sum_{l, m=1}^{\nu_{1}}\left|p_{l}\right\rangle\left(\Delta^{-1}\right)_{l m}\left\langle\bar{p}_{m}\right|
$$

with $\Delta_{l m}=\left\langle\bar{p}_{l} \mid p_{m}\right\rangle$. Here $\left\langle\bar{p}_{l}\right| \Phi_{-}^{-1}\left(\bar{k}_{1}\right)=0$ and $\Phi_{+}\left(k_{1}\right)\left|p_{l}\right\rangle=0$. In the particular case of $n=N-1$ and the Hermitian potential we get the vector soliton solution, for which

$$
P_{r}=\frac{|p\rangle\langle p|}{\langle p \mid p\rangle}, \quad|p\rangle=\exp \left\{i k_{1} x A\right\}|P\rangle
$$

Define complex parameters $C_{l}=P_{l} / P_{N}$. The $t$-dependence of $C_{l}$ then follows from equation (35). It can be accounted for in a convenient way by introducing real $t$-dependent parameters $\bar{x}$, and $\delta_{l}, l=1, \ldots, n$, the soliton position and phases of its components. Let $C_{l}=\theta_{l} e^{2(\eta-i \xi) \bar{x}}$, where $\theta_{l}=s_{l} e^{i \delta_{l}}$. The amplitudes $s_{l}$, satisfying

$$
\sum_{l=1}^{n} s_{l}^{2}=1
$$

describe polarization of the multi-component soliton. Integrating equation (35) we obtain

$$
\bar{x}=\bar{x}_{0}+\frac{\operatorname{Im}\{\omega(i \eta+\xi)\} t}{\eta}, \quad \delta_{l}=\delta_{l 0}+\frac{2}{\eta} \operatorname{Im}\{(\xi-i \eta) \omega(i \eta+\xi)\} t .
$$

The vector soliton then takes the form

$$
q_{l}=2 i \eta \theta_{l} e^{i(\xi / \eta) z} \operatorname{sech} z
$$

with $z=2 \eta(x-\bar{x})$. For instance, for the vector NLS equation $\omega(k)=2 k^{2}$ and we arrive at the $n$-component generalization of the well-known vector soliton solution for the two-component NLS equation [12].

\section{PERTURBATION-INDUCED EVOLUTION OF THE SPECTRAL DATA}

A perturbation of the integrable PDE, following from the Lax representation (1)-(2), can be written in the form

$$
i Q_{t}-V_{x}+[i k A+i Q, V]=R
$$


where $R$ (which is $k$-independent in case of the Zakharov-Shabat spectral problem) represents the terms destroying integrablity. The perturbation matrix $R$, similar to the potential $Q$, satisfies $[A, R]=0$. For the Hermitian potential $Q^{\dagger}=Q$ we get also $R^{\dagger}=-R$.

Below we derive evolution equations for the spectral data with account for arbitrary perturbation. For simplicity of the presentation we will frequently omit the explicit dependence on $x$ and $t$. To distinguish between the "integrable" and "perturbation" contributions to the evolution let us assign the variational derivatives to the latter. For instance, the perturbation-induced evolution in equation (41) reads

$$
i \frac{\delta Q}{\delta t}=R, \quad \text { or, explicitly } \quad i \frac{\delta \mathbf{q}}{\delta t}=\mathbf{r} \quad \text { and } \quad i \frac{\delta \overline{\mathbf{q}}}{\delta t}=-\overline{\mathbf{r}}
$$

where $\mathbf{r}=R_{I, I I}$ and $\overline{\mathbf{r}}=-R_{I I, I}$. For Hermitian $Q, \overline{\mathbf{r}}=\mathbf{r}^{\dagger}$.

We start with derivation of evolution equations for $\Phi_{+}$and $\Phi_{-}^{-1}$, the solution to the RH problem (23). In other terms, we are going to derive the generalized Lax pair (see equations (52) and (53) below) for the perturbed nonlinear PDE (41). Differentiation of equation (11) with respect to $t$ gives

$$
\partial_{x}\left(\frac{\delta \Phi}{\delta t}\right)=i k\left[A, \frac{\delta \Phi}{\delta t}\right]+i Q \frac{\delta \Phi}{\delta t}+R \Phi
$$

and consequently

$$
\partial_{x}\left(\Phi^{-1} \frac{\delta \Phi}{\delta t}\right)=i k\left[A, \Phi^{-1} \frac{\delta \Phi}{\delta t}\right]+\Phi^{-1} R \Phi
$$

Let us integrate the above formula for $\Phi=J_{ \pm}$. We get

$$
\frac{\delta J_{ \pm}}{\delta t}(x, k)=J_{ \pm}(x, k) e^{i k x A}\left(\int_{ \pm \infty}^{x} \mathrm{~d} \xi e^{-i k \xi A} J_{ \pm}^{-1}(\xi, k) R(\xi) J_{ \pm}(\xi, k) e^{i k \xi A}\right) e^{-i k x A}
$$

Here we have used that $\delta J_{ \pm} \rightarrow 0$ as $x \rightarrow \pm \infty$. Let us employ the relation between $J_{ \pm}$and $\Phi_{+}$to rewrite this formula in a more convenient form. Using (20a) we get

$$
\frac{\delta J_{ \pm}}{\delta t}(x, k)=J_{ \pm}(x, k) e^{i k x A} S_{ \pm}(k) \Upsilon( \pm \infty, x ; k) S_{ \pm}^{-1}(k) e^{-i k x A}
$$

In much the same way, using (20b), we derive 


$$
\frac{\delta J_{ \pm}^{-1}}{\delta t}(x, k)=-e^{i k x A} \bar{S}_{ \pm}^{-1}(k) \bar{\Upsilon}( \pm \infty, x ; k) \bar{S}_{ \pm}(k) e^{-i k x A} J_{ \pm}^{-1}(x, k)
$$

Here we have introduced the notations:

$$
\begin{aligned}
& \Upsilon( \pm \infty, x ; k)=\int_{ \pm \infty}^{x} \mathrm{~d} \xi e^{-i k \xi A} \Phi_{+}^{-1} R \Phi_{+} e^{i k \xi A} \\
& \bar{\Upsilon}( \pm \infty, x ; k)=\int_{ \pm \infty}^{x} \mathrm{~d} \xi e^{-i k \xi A} \Phi_{-}^{-1} R \Phi_{-} e^{i k \xi A} .
\end{aligned}
$$

These matrix functionals will enter every formula describing perturbation-induced evolution of the spectral data. For the involution, due to formula (19) these matrix functionals satisfy

$$
\Upsilon^{\dagger}( \pm \infty, x ; k)=-\bar{\Upsilon}( \pm \infty, x, \bar{k})
$$

Using equations (42), (43), and the definition of the scattering matrix $S$ (14) we get

$$
\begin{gathered}
\frac{\delta S}{\delta t}=e^{-i k x A} \frac{\delta}{\delta t}\left(J_{+}^{-1} J_{-}\right) e^{i k x A}=-\bar{S}_{+}^{-1} \bar{\Upsilon}(\infty, x) \bar{S}_{+} S+S S_{-} \Upsilon(-\infty, x) S_{-}^{-1} \\
=S_{+} \Upsilon(-\infty, x) S_{-}^{-1}+\bar{S}_{+}^{-1} \bar{\Upsilon}(x, \infty) \bar{S}_{-} .
\end{gathered}
$$

Setting $x \rightarrow \pm \infty$ in this formula produces two simple equivalent formulae:

$$
\frac{\delta S(k)}{\delta t}=S_{+}(k) \Upsilon(-\infty, \infty ; k) S_{-}^{-1}(k) \equiv \bar{S}_{+}^{-1}(k) \bar{\Upsilon}(-\infty, \infty ; k) \bar{S}_{-}(k)
$$

Now we can easily obtain the perturbation-induced evolution of the solution to the RH problem. Taking into account the definitions of $S_{ \pm}$and $\bar{S}_{ \pm}$and (20) we write:

$$
\begin{gathered}
\frac{\delta \Phi_{+}}{\delta t}=\frac{\delta J_{+}}{\delta t} e^{i k x A} S_{+} e^{-i k x A}+J_{+} e^{i k x A} \frac{\delta}{\delta t}\left(H_{1}+S H_{2}\right) e^{-i k x A} \\
=\Phi_{+} e^{i k x A}\left(-\Upsilon(x, \infty)+\Upsilon(-\infty, \infty) H_{2}\right) e^{-i k x A}=\Phi_{+} e^{i k x A} \Pi e^{-i k x A} \\
\frac{\delta \Phi_{-}^{-1}}{\delta t}=e^{i k x A} \bar{S}_{+} e^{-i k x A} \frac{\delta J_{+}^{-1}}{\delta t}+e^{i k x A} \frac{\delta}{\delta t}\left(H_{1}+H_{2} S^{-1}\right) J_{+}^{-1} e^{-i k x A} \\
=e^{i k x A}\left(\bar{\Upsilon}(x, \infty)-H_{2} \bar{\Upsilon}(-\infty, \infty)\right) \Phi_{-}^{-1} e^{-i k x A}=-e^{i k x A} \bar{\Pi} \Phi_{-}^{-1} e^{-i k x A}
\end{gathered}
$$


The r.h.s.'s of the above formulae contain the evolution functionals,

$$
\begin{gathered}
\Pi(x, k)=-\Upsilon(x, \infty ; k) H_{1}+\Upsilon(-\infty, x ; k) H_{2}, \\
\bar{\Pi}(x, k)=H_{1} \bar{\Upsilon}(x, \infty ; k)-H_{2} \bar{\Upsilon}(-\infty, x ; k),
\end{gathered}
$$

which account for the perturbation-induced evolution of the solution to the RH problem. As follows from formula (46), for the case of the involution the evolution functionals satisfy

$$
\Pi^{\dagger}(k)=-\bar{\Pi}(\bar{k})
$$

From the definitions (49), (50) and also (44), (45) it is easy to see that the matrices $e^{i k x A} \Pi(k) e^{-i k x A}$ and $e^{i k x A} \bar{\Pi}(k) e^{-i k x A}$ are meromorphic and bounded in the upper and lower half planes of the $k$-plane, respectively. They have simple poles respectively at zeros of $\operatorname{det} \Phi_{+}(k)$ and $\operatorname{det} \Phi_{-}^{-1}(k)$.

Let us write down the generalized Lax representation for the perturbed PDE (41) in terms of $\Phi_{+}(x, k)$ :

$$
\begin{gathered}
\partial_{x} \Phi_{+}=i k\left[A, \Phi_{+}\right]+i Q \Phi_{+}, \\
\partial_{t} \Phi_{+}=\Phi_{+} \Omega+V \Phi_{+}+\Phi_{+} e^{i k x A} \Pi e^{-i k x A} .
\end{gathered}
$$

It is easy to check by direct calculation that the compatibility condition for the above linear system is equivalent to the perturbed equation (41) (indeed, $\partial_{x} \Pi=e^{-i k x A} \Phi_{+}^{-1} R \Phi_{+} e^{i k x A}$ ).

Now, let us derive the perturbation-induced evolution of the spectral data. From (48) we immediately obtain

$$
\frac{\delta G}{\delta t}=\frac{\delta}{\delta t}\left(e^{-i k x A} \Phi_{-}^{-1} \Phi_{+} e^{i k x A}\right)=G \Pi-\bar{\Pi} G
$$

Hence, the complete evolution of the continuous datum reads

$$
\partial_{t} G=[G, \Omega]+G \Pi-\bar{\Pi} G
$$


where we have taken into account the integrable evolution given by (32). It is important to notice that the l.h.s. of (54) does not depend on $x$. Therefore, we can put $x \rightarrow \pm \infty$ in this equation to simplify it. Equation (54) can be rewritten in a more explicit form. Using the definition (24), formulae (49) and (50) we get two equivalent evolution equations for $\mathbf{b}$ corresponding to the two limits $x \rightarrow \pm \infty$ :

$$
\begin{aligned}
& \partial_{t} \mathbf{b}=-2 i \omega(k) \mathbf{b}+\mathbf{b} H_{2} \Upsilon(-\infty, \infty ; k) H_{2}+H_{1} \Upsilon(-\infty, \infty ; k) H_{2}, \\
& \partial_{t} \mathbf{b}=-2 i \omega(k) \mathbf{b}-H_{1} \bar{\Upsilon}(-\infty, \infty ; k) H_{1} \mathbf{b}-H_{1} \bar{\Upsilon}(-\infty, \infty ; k) H_{2} .
\end{aligned}
$$

Similarly,

$$
\begin{aligned}
& \partial_{t} \overline{\mathbf{b}}=2 i \omega(k) \overline{\mathbf{b}}+\overline{\mathbf{b}} H_{1} \Upsilon(-\infty, \infty ; k) H_{1}+H_{2} \Upsilon(-\infty, \infty ; k) H_{1}, \\
& \partial_{t} \overline{\mathbf{b}}=2 i \omega(k) \overline{\mathbf{b}}-H_{2} \bar{\Upsilon}(-\infty, \infty ; k) H_{2} \overline{\mathbf{b}}-H_{2} \bar{\Upsilon}(-\infty, \infty ; k) H_{1} .
\end{aligned}
$$

Evolution of zeros $k_{j}$ and $\bar{k}_{j}$ of $\operatorname{det} \Phi_{+}(k)$ and $\operatorname{det} \Phi_{-}^{-1}(k)$, respectively, is derived by differentiation of the determinants. In the integrable limit zeros do not depend on $t$. Then, for instance,

$$
\frac{\mathrm{d} k_{j}}{\mathrm{~d} t}=-\left.\frac{\partial_{t} \operatorname{det} \Phi_{+}(k)}{\partial_{k} \operatorname{det} \Phi_{+}(k)}\right|_{k=k_{j}}=-\left.\frac{\operatorname{Tr}\{\Pi(k)\} \operatorname{det} \Phi_{+}(k)}{\partial_{k} \operatorname{det} \Phi_{+}(k)}\right|_{k=k_{j}} .
$$

To calculate the r.h.s. in this formula recall that the evolution functional $\Pi$ has simple pole at $k=k_{j}$ and that $\operatorname{det} \Phi_{+}(k)$ can be written as

$$
\operatorname{det} \Phi_{+}(k)=\operatorname{det} \phi_{+}(k) \operatorname{det} \Gamma(k)=\operatorname{det} \phi_{+}(k) \prod_{i=1}^{s}\left(\frac{k-k_{i}}{k-\bar{k}_{i}}\right)^{\nu_{i}}
$$

where $\operatorname{det} \phi_{+}(k) \neq 0$. Simple calculations give

$$
\frac{\mathrm{d} k_{j}}{\mathrm{~d} t}=-\frac{\operatorname{Tr}\left\{\operatorname{Res} \Pi\left(k_{j}\right)\right\}}{\nu_{j}}
$$

Here "Res" denotes the residue of $\Pi(k)$ at $k=k_{j}$. Noticing that the 1.h.s. does not depend on $x$, we simplify the above equation setting $x \rightarrow \pm \infty$ : 


$$
\frac{\mathrm{d} k_{j}}{\mathrm{~d} t}=-\frac{\operatorname{Tr}\left\{\operatorname{Res} \Upsilon\left(-\infty, \infty ; k_{j}\right) H_{2}\right\}}{\nu_{j}} \equiv \frac{\operatorname{Tr}\left\{\operatorname{Res} \Upsilon\left(-\infty, \infty ; k_{j}\right) H_{1}\right\}}{\nu_{j}}
$$

Similarly, using $\operatorname{det} \Phi_{-}^{-1}$, we get

$$
\frac{\mathrm{d} \bar{k}_{j}}{\mathrm{~d} t}=-\frac{\operatorname{Tr}\left\{\operatorname{Res} \bar{\Upsilon}\left(-\infty, \infty ; \bar{k}_{j}\right) H_{2}\right\}}{\nu_{j}} \equiv \frac{\operatorname{Tr}\left\{\operatorname{Res} \bar{\Upsilon}\left(-\infty, \infty ; \bar{k}_{j}\right) H_{1}\right\}}{\nu_{j}}
$$

In derivation of the perturbation-induced evolution of the null vectors we will use the following remarkable identities (written for the $x$-independent null vectors, see (37))

$$
\operatorname{Res} \Pi\left(k_{j}\right)\left|P_{l}^{(j)}\right\rangle=-\frac{\delta k_{j}}{\delta t}\left|P_{l}^{(j)}\right\rangle, \quad\left\langle\bar{P}_{l}^{(j)}\right| \operatorname{Res} \bar{\Pi}\left(\bar{k}_{j}\right)=\left\langle\bar{P}_{l}^{(j)}\right| \frac{\delta \bar{k}_{j}}{\delta t}
$$

as well as other two identities:

$$
\Phi_{+}\left(k_{j}\right) e^{i k_{j} x A} \operatorname{Res} \Pi\left(k_{j}\right)=0, \quad \operatorname{Res} \bar{\Pi}\left(\bar{k}_{j}\right) e^{-i \bar{k}_{j} x A} \Phi_{-}^{-1}\left(\bar{k}_{j}\right)=0
$$

which follow from (48). To verify the identities (59) one can proceed as follows. Introduce functions $F_{j}^{(+)}(k)=\left(k-k_{j}\right) \Phi_{+}^{-1}(k)$ and $F_{j}^{(-)}(k)=\left(k-\bar{k}_{j}\right) \Phi_{-}(k)$. The regularization matrices $\Gamma^{-1}(k)$ and $\Gamma(k)$ have simple poles at $k=k_{j}$ and $k=\bar{k}_{j}$, respectively. Hence the introduced matrix functions are holomorphic in some neighborhoods of these points. Now compute the product

$$
\begin{gathered}
\left\{F_{j}^{(+)}(k) \frac{\delta \Phi_{+}(k)}{\delta t}\left|p_{l}^{(j)}\right\rangle\right\}_{k=k_{j}}=\left\{\left(k-k_{j}\right) e^{i k x A} \Pi(k) e^{-i k x A}\left|p_{l}^{(j)}\right\rangle\right\}_{k=k_{j}} \\
=e^{i k_{j} x A} \operatorname{Res} \Pi\left(k_{j}\right) e^{-i k_{j} x A}\left|p_{l}^{(j)}\right\rangle
\end{gathered}
$$

On the other hand,

$$
\begin{aligned}
\left\{F_{j}^{(+)}(k) \frac{\delta \Phi_{+}(k)}{\delta t}\right\}_{k=k_{j}} & =\left\{\frac{\delta}{\delta t}\left(k-k_{j}\right) I\right\}_{k=k_{j}}-\left\{\frac{\delta F_{j}^{(+)}(k)}{\delta t} \Phi_{+}(k)\right\}_{k=k_{j}} \\
& =-\frac{\delta k_{j}}{\delta t} I-\frac{\delta F_{j}^{(+)}\left(k_{j}\right)}{\delta t} \Phi_{+}\left(k_{j}\right) .
\end{aligned}
$$

Multiplication by $\left|p_{l}^{(j)}\right\rangle$ of the latter formula and comparison with the former leads to the first identity in (59). The second one can be checked in similar way. 
Now let us derive evolution equations for the $x$-independent null vectors defined in (37). To this goal we simply differentiate equation (26)

$$
\begin{gathered}
\frac{\delta}{\delta t}\left(\Phi_{+}\left(k_{j}\right) e^{i k_{j} x A}\left|P_{l}^{(j)}\right\rangle\right)=\left\{\Phi_{+}(k) e^{i k x A} \Pi(k)\left|P_{l}^{(j)}\right\rangle\right\}_{k=k_{j}}+\frac{\delta k_{j}}{\delta t} \frac{\partial \Phi_{+}\left(k_{j}\right)}{\partial k} e^{i k_{j} x A}\left|P_{l}^{(j)}\right\rangle \\
+\frac{\delta k_{j}}{\delta t} \Phi_{+}\left(k_{j}\right) i x A e^{i k_{j} x A}\left|P_{l}^{(j)}\right\rangle+\Phi_{+}\left(k_{j}\right) e^{i k_{j} x A} \frac{\delta\left|P_{l}^{(j)}\right\rangle}{\delta t}=0 .
\end{gathered}
$$

Denote $\Pi_{r}\left(k_{j}\right)$ the value of the regular part of $\Pi(k)$ at $k=k_{j}$,

$$
\Pi_{r}\left(k_{j}\right)=\left\{\Pi(k)-\frac{\operatorname{Res} \Pi\left(k_{j}\right)}{k-k_{j}}\right\}_{k=k_{j}} .
$$

Then, using (59) and (60) to cancel out secular terms, we arrive at

$$
\Phi_{+}\left(k_{j}\right) e^{i k_{j} x A}\left\{\frac{\delta\left|P_{l}^{(j)}\right\rangle}{\delta t}+\Pi_{r}\left(k_{j}\right)\left|P_{l}^{(j)}\right\rangle\right\}=0 .
$$

Using the same arguments as in section III for derivation of the integrable evolution we get, adding the latter,

$$
\frac{\mathrm{d}\left|P_{l}^{(j)}\right\rangle}{\mathrm{d} t}=-\Omega\left(k_{j}\right)\left|P_{l}^{(j)}\right\rangle-\Pi_{r}\left(k_{j}\right)\left|P_{l}^{(j)}\right\rangle .
$$

Similarly,

$$
\frac{\mathrm{d}\left\langle\bar{P}_{l}^{(j)}\right|}{\mathrm{d} t}=\left\langle\bar{P}_{l}^{(j)}\right| \Omega\left(\bar{k}_{j}\right)+\left\langle\bar{P}_{l}^{(j)}\right| \bar{\Pi}_{r}\left(\bar{k}_{j}\right),
$$

where

$$
\bar{\Pi}_{r}\left(\bar{k}_{j}\right)=\left\{\bar{\Pi}(k)-\frac{\operatorname{Res} \bar{\Pi}\left(k_{j}\right)}{k-\bar{k}_{j}}\right\}_{k=\bar{k}_{j}} .
$$

In the case of the involution equation (63) is Hermitian conjugate to (62). Note that the l.h.s.'s of equations (62) and (63) do not depend on $x$. Hence we can sent $x \rightarrow \pm \infty$ to considerably simplify these equations. Consider, for instance, (62). Letting $x \rightarrow \infty$ and introducing the notations

$$
\left|P_{l}^{(j)}\right\rangle=H_{1}\left|P_{l}^{(j)}\right\rangle+H_{2}\left|P_{l}^{(j)}\right\rangle \equiv\left|P_{l}^{(j)}, 1\right\rangle+\left|P_{l}^{(j)}, 2\right\rangle
$$


and $\Upsilon^{(1)}=H_{1} \Upsilon H_{2}, \Upsilon^{(2)}=H_{2} \Upsilon H_{2}$ we get the following system

$$
\begin{gathered}
\frac{\mathrm{d}\left|P_{l}^{(j)}, 1\right\rangle}{\mathrm{d} t}=-i \omega\left(k_{j}\right)\left|P_{l}^{(j)}, 1\right\rangle-\Upsilon_{r}^{(1)}\left(-\infty, \infty ; k_{j}\right)\left|P_{l}^{(j)}, 2\right\rangle \\
\frac{\mathrm{d}\left|P_{l}^{(j)}, 2\right\rangle}{\mathrm{d} t}=\left\{i \omega\left(k_{j}\right)-\Upsilon_{r}^{(2)}\left(-\infty, \infty ; k_{j}\right)\right\}\left|P_{l}^{(j)}, 2\right\rangle .
\end{gathered}
$$

Here $\Upsilon_{r}\left(k_{j}\right)$ is value of the regular part of the matrix $\Upsilon(k)$ at $k=k_{j}$ :

$$
\Upsilon_{r}\left(k_{j}\right)=\left\{\Upsilon(k)-\frac{\operatorname{Res} \Upsilon\left(k_{j}\right)}{k-k_{j}}\right\}_{k=k_{j}}
$$

When dealing with vector PDEs, i.e., for $n=N-1$, one can define the polarization-phase parameters of vector solitons as quotients of components of the null vectors (note that the zeros are simple in this particular case). Let $C_{l}^{(j)}=P_{l}^{(j)} / P_{N}^{(j)}, l=1, \ldots, n=N-1$, where $\left|P^{(j)}\right\rangle=\left(P_{1}^{(j)}, \ldots, P_{N}^{(j)}\right)^{T}$. Then from (65) we obtain:

$$
\frac{\mathrm{d} C_{l}^{(j)}}{\mathrm{d} t}=\left\{-2 i \omega\left(k_{j}\right)+\Upsilon_{r N N}\left(-\infty, \infty ; k_{j}\right)\right\} C_{l}^{(j)}-\Upsilon_{r l N}\left(-\infty, \infty ; k_{j}\right), \quad l=1, \ldots, n .
$$

This particular case $(n=N-1)$ contains the vector NLS (8) and the complex modified $\mathrm{KdV}$ (9) equations as examples. In view of considerable importance of such vector nonlinear PDEs, we formulate the result of this section in the following theorem.

\section{Theorem: Let}

$$
i Q_{t}+V_{0}\left(Q, Q_{x}, Q_{x x}, \ldots\right)=\epsilon R\left(x, t, Q, Q_{x}, Q_{x x}, \ldots\right)
$$

be a perturbed nonlinear PDE associated with the $N \times N$ matrix Zakharov-Shabat spectral problem,

$$
\partial_{x} \Phi=i k[A, \Phi]+i Q(x, t) \Phi, \quad A=\operatorname{diag}\left(I_{n},-1\right)
$$

where $(n=N-1)$

$$
Q=\left(\begin{array}{cc}
0 & \mathbf{q} \\
\mathbf{q}^{\dagger} & 0
\end{array}\right), \quad R=\left(\begin{array}{cc}
0 & \mathbf{r} \\
-\mathbf{r}^{\dagger} & 0
\end{array}\right), \quad \mathbf{q}=\left(q_{1}, q_{2}, \ldots, q_{n}\right)^{T}, \quad \mathbf{r}=\left(r_{1}, r_{2}, \ldots, r_{n}\right)^{T}
$$


Here the matrix function $V_{0}$ represents the limiting integrable evolution given by the dispersion relation $\Omega(k)=i \omega(k) A$, while $R$ contains the terms destroying integrability ( $\epsilon$ is a small parameter). Then the perturbed evolution is equivalent to the following evolution of the spectral data:

$$
\begin{gathered}
\frac{\mathrm{d} k_{j}}{\mathrm{~d} t}=-\epsilon \operatorname{Res} \Upsilon_{N N}\left(t, k_{j}\right), \\
\frac{\mathrm{d} C_{l}^{(j)}}{\mathrm{d} t}=\left\{-2 i \omega\left(k_{j}\right)+\epsilon \Upsilon_{r N N}\left(t, k_{j}\right)\right\} C_{l}^{(j)}-\epsilon \Upsilon_{r l N}\left(t, k_{j}\right), \quad l=1, \ldots, n, \\
\frac{\partial b_{l}(k)}{\partial t}=\left\{-2 i \omega(k)+\epsilon \Upsilon_{N N}(t, k)\right\} b_{l}(k)+\epsilon \Upsilon_{l N}(t, k), \quad l=1, \ldots, n .
\end{gathered}
$$

Here

$$
\begin{gathered}
\Upsilon(t, k)=\int_{-\infty}^{\infty} \mathrm{d} x e^{-i k x A} \Phi_{+}^{-1}(x, t, k) R(x, t) \Phi_{+}(x, t, k) e^{i k x A}, \\
\Upsilon_{r}\left(t, k_{j}\right)=\left\{\Upsilon(t, k)-\frac{\operatorname{Res} \Upsilon\left(t, k_{j}\right)}{k-k_{j}}\right\}_{k=k_{j}} .
\end{gathered}
$$

The matrix function $\Phi_{+}(x, t, k)$ solves the spectral problem (68) and the $R H$ problem

$$
\begin{gathered}
\Phi_{+}^{\dagger}(x, t, k) \Phi_{+}(x, t, k)=e^{i k x A} G(k, t) e^{-i k x A}, \quad k \in \mathrm{Re}, \\
\Phi_{+}(k) \rightarrow I, \quad k \rightarrow \infty
\end{gathered}
$$

of analytic factorization of matrix $G(k, t)$,

$$
G(k, t)=\left(\begin{array}{cc}
I_{n} & \mathbf{b}(k, t) \\
\mathbf{b}^{\dagger}(k, t) & 1
\end{array}\right), \quad \mathbf{b}=\left(b_{1}, b_{2}, \ldots, b_{n}\right)^{T}
$$

The Riemann-Hilbert problem has (simple) zeros $k_{j}(t), j=1, \ldots, s$, given by $a\left(k_{j}, t\right)=$ $\operatorname{det} \Phi_{+}\left(x, t, k_{j}\right)=0$. The vector-columns $\left|C^{(j)}(t)\right\rangle=\left(C_{1}^{(j)}(t), C_{2}^{(j)}(t), \ldots, C_{n}^{(j)}(t), 1\right)$ are the null vectors of the matrices $\Phi_{+}\left(x, t, k_{j}\right) e^{i k_{j} x A}$ :

$$
\Phi_{+}\left(x, t, k_{j}\right) e^{i k_{j} x A}\left|C^{(j)}(t)\right\rangle=0, \quad j=1, \ldots, s .
$$


The initial spectral data are obtained via solution of the spectral equation (68) and represent the spectral characterization of the potential $Q(x, 0)$. For real $k$, the spectral densities of radiation $b_{l}(k, t)$ and the function $a(k, t)$ satisfy the following identity

$$
\bar{a} a=1-\sum_{l=1}^{n} \bar{b}_{l} b_{l}
$$

The potential $\mathbf{q}(x, t)$ is reconstructed by the formula

$$
q_{l}(x, t)=2 \lim _{k \rightarrow \infty} k\left(\Phi_{+}\right)_{l, N}(x, t, k), \quad l=1, \ldots, n .
$$

Some comments are necessary on the use of equations (69)-(71) for the spectral data. These equations are exact, i.e., they account for the perturbation exactly. As a consequence, for the non-integrable PDE (67), these equations are non-closed ODEs. Equations (69)-(71) are non-closed because they contain explicitly the matrix $\Phi_{+}(x, t, k)$, solution of the $\mathrm{RH}$ problem, obtaining which requires knowledge of the spectral data. Therefore, equations (69)-(71) serve as the generating equations for the perturbation expansion: expanding the spectral data into the asymptotic power series in $\epsilon$, one obtains the sequence of closed approximate ODEs for the spectral data. On this way, one does not need to solve the $\mathrm{RH}$ problem - the computations are algebraic. In the next section we consider a single multi-component soliton as an example.

\section{MULTI-COMPONENT SOLITON UNDER PERTURBATIONS}

In this section we apply the theorem for construction of the perturbation theory for a single multi-component soliton. This can be done without specifying the dispersion relation $\omega(k)$ determining the evolution of the spectral data in the unperturbed PDE. Hence our results apply to all nearly integrable vector PDEs associated with the Zakharov-Shabat spectral problem. Below we derive equations describing the evolution of the soliton param-

eters and give formulae for the first-order radiation. For a single vector soliton given by formula (40), i.e., $q_{l}=2 i \eta \theta_{l} e^{i(\xi / \eta) z} \operatorname{sech} z$, where $z=2 \eta(x-\bar{x})$, the regularization matrix $\Gamma$ has the form 


$$
\Gamma=I-\frac{i \eta}{\left(k-\bar{k}_{1}\right) \cosh z}\left(\begin{array}{cc}
e^{-z}|\theta\rangle\langle\theta| & e^{i(\xi / \eta) z}|\theta\rangle \\
\langle\theta| e^{-i(\xi / \eta) z} & e^{z}
\end{array}\right)
$$

Here $k_{1}=\xi+i \eta$ and we have used the vector notations $|\theta\rangle=\left(\theta_{1}, \ldots, \theta_{n}\right)^{T}$ and $\langle\theta|=\left(\bar{\theta}_{1}, \ldots \bar{\theta}_{n}\right)$. To simplify some of the calculations introduce the following basis (in the $n$-dimensional subspace)

$$
\left|\theta^{(1)}\right\rangle=|\theta\rangle, \quad\left|\theta^{(2)}\right\rangle, \quad \ldots, \quad\left|\theta^{(n)}\right\rangle ; \quad\left\langle\theta^{(l)} \mid \theta^{(m)}\right\rangle=\delta_{l m}
$$

The explicit form of the vectors $\left|\theta^{(l)}\right\rangle$ for $l=2, \ldots, n$ will not be needed at all. Also we will use the basis

$$
\left|e_{1}\right\rangle=(1,0, \ldots, 0)^{T}, \quad\left|e_{2}\right\rangle=(0,1,0, \ldots, 0)^{T}, \quad \ldots, \quad\left|e_{n}\right\rangle=(0, \ldots, 0,1)^{T}
$$

With the help of the unitary transformation matrix $\Xi$, defined as

$$
\Xi=\left(\begin{array}{cc}
B & 0 \\
0 & 1
\end{array}\right), \quad B=\sum_{l=1}^{n}\left|e_{l}\right\rangle\left\langle\theta^{(l)}\right|,
$$

the regularization matrix $\Gamma$ can be considerably simplified:

$$
\widetilde{\Gamma}=\Xi \Gamma \Xi^{-1}=I-\frac{i \eta}{\left(k-\bar{k}_{1}\right) \cosh z}\left(\begin{array}{cc}
e^{-z}\left|e_{1}\right\rangle\left\langle e_{1}\right| & e^{i(\xi / \eta) z}\left|e_{1}\right\rangle \\
\left\langle e_{1}\right| e^{-i(\xi / \eta) z} & e^{z}
\end{array}\right)
$$

Note the evident property $\widetilde{\Gamma}^{-1}=\widetilde{\Gamma}^{\dagger}$.

The transformation matrix $\Xi$ depends on $t$ due to the perturbation-induced time dependence of the $\theta$-parameters, but does not depend on $x$. This simple fact allows us to use the transformation with $\Xi$ inside the integrals defining $\Upsilon(\sqrt{72})$. Taking into account that $\Phi_{+}=\Gamma$ for the pure soliton solution of the unperturbed PDE, in the first order we get

$$
\widetilde{\Upsilon}=\Xi \Upsilon \Xi^{-1}=\int_{-\infty}^{\infty} \mathrm{d} x e^{-i k x A} \widetilde{\Gamma}^{-1} \widetilde{R} \widetilde{\Gamma} e^{i k x A}
$$

Here we have defined

$$
\widetilde{R}=\Xi R \Xi^{-1}=\left(\begin{array}{cc}
0 & B|r\rangle \\
-\langle r| B^{\dagger} & 0
\end{array}\right)
$$


where, for convenience of the presentation below, we have changed the notation for the perturbation: $|r\rangle=\mathbf{r}=\left(r_{1}, \ldots, r_{n}\right)^{T}$.

In fact, we will need only one diagonal element

$$
\Upsilon_{N N}=\left(\Xi^{-1} \widetilde{\Upsilon} \Xi\right)_{N N}=\int_{-\infty}^{\infty} \mathrm{d} x\left(\widetilde{\Gamma}^{\dagger} \widetilde{R} \widetilde{\Gamma}\right)_{N N}
$$

and the following non-diagonal matrix elements

$$
\Upsilon_{l N}=\left(\Xi^{-1} \widetilde{\Upsilon} \Xi\right)_{l N}=\sum_{m=1}^{n} B_{l m}^{-1} \widetilde{\Upsilon}_{m N}=\theta_{l} \widetilde{\Upsilon}_{1 N}+\sum_{m=2}^{n} B_{l m}^{-1} \widetilde{\Upsilon}_{m N}=\theta_{l} \widetilde{\Upsilon}_{1 N}+F_{l}
$$

where $l=1, \ldots, n$. The second term simplifies as follows

$$
\begin{gathered}
F_{l}=\int_{-\infty}^{\infty} \mathrm{d} x e^{-2 i k x} \sum_{m=2}^{n} B_{l m}^{-1} \widetilde{R}_{m N} \widetilde{\Gamma}_{N N}=\int_{-\infty}^{\infty} \mathrm{d} x e^{-2 i k x} \widetilde{\Gamma}_{N N} \sum_{m=2}^{n}\left\langle e_{l}\left|B^{-1}\right| e_{m}\right\rangle\left\langle e_{m}|B| r\right\rangle \\
=\int_{-\infty}^{\infty} \mathrm{d} x e^{-2 i k x} \widetilde{\Gamma}_{N N}\left(r_{l}-\theta_{l}\langle\theta \mid r\rangle\right) .
\end{gathered}
$$

After simple calculations we get:

$$
\begin{gathered}
\Upsilon_{N N}=\frac{i}{4} \int_{-\infty}^{\infty} \mathrm{d} z \operatorname{sech}^{2} z\left(\frac{e^{-z}}{k-k_{1}}+\frac{e^{z}}{k-\bar{k}_{1}}\right)\left(r_{0}(z)+\bar{r}_{0}(-z)\right), \\
\Upsilon_{l N}=\frac{\theta_{l}}{8 \eta} \int_{-\infty}^{\infty} \mathrm{d} z \operatorname{sech}^{2} z \frac{\exp \{-2 i k x+i(\xi / \eta) z\}}{\left(k-k_{1}\right)\left(k-\bar{k}_{1}\right)}\left\{-4 \eta^{2} \bar{r}_{0}(-z)\right. \\
\left.+\left[e^{-2 z}\left(k-\bar{k}_{1}\right)+e^{2 z}\left(k-k_{1}\right)\right]^{2} r_{0}(z)\right\}+F_{l} .
\end{gathered}
$$

Here we have used the notation

$$
r_{0}=e^{-i(\xi / \eta) z} \widetilde{R}_{1 N}=e^{-i(\xi / \eta) z}\langle\theta \mid r\rangle=e^{-i(\xi / \eta) z} \sum_{l=1}^{n} \bar{\theta}_{l} r_{l} .
$$




\section{A. Evolution of the soliton parameters}

Let us first derive evolution equations for the soliton parameters $\eta, \xi$, $\bar{x}$, and $\theta_{l}, l=$ $1, \ldots, n$. Using the identity $-2 i k_{1} x+i(\xi / \eta) z=z+2 \eta \bar{x}-2 i \xi \bar{x}$ and the definition $C_{l}=\theta_{l} e^{2(\eta-i \xi) \bar{x}}$ from section [II], we obtain:

$$
\begin{gathered}
\operatorname{Res}_{N N}\left(k_{1}\right)=\frac{i}{4} \int_{-\infty}^{\infty} \mathrm{d} z e^{-z} \operatorname{sech}^{2} z\left(r_{0}(z)+\bar{r}_{0}(-z)\right), \\
\Upsilon_{r N N}\left(k_{1}\right)=\frac{1}{8 \eta} \int_{-\infty}^{\infty} \mathrm{d} z e^{z} \operatorname{sech}^{2} z\left(r_{0}(z)+\bar{r}_{0}(-z)\right), \\
\Upsilon_{r l N}\left(k_{1}\right)=C_{l}\left\{-2 i \bar{x} \operatorname{Res}\left\{\Upsilon_{N N}\left(k_{1}\right)\right\}+\Upsilon_{r N N}\left(k_{1}\right)+J_{0}\right\}+f_{l},
\end{gathered}
$$

where

$$
J_{0}=\frac{1}{4 \eta} \int_{-\infty}^{\infty} \mathrm{d} z \operatorname{sech}^{2} z\left(\cosh z+z e^{-z}\right)\left(r_{0}(z)-\bar{r}_{0}(-z)\right)
$$

and

$$
f_{l}=F_{l}\left(k_{1}\right)=\frac{e^{2(\eta-i \xi) \bar{x}}}{4 \eta} \int_{-\infty}^{\infty} \mathrm{d} z \operatorname{sech} z\left(e^{-i(\xi / \eta) z} r_{l}-\theta_{l} r_{0}\right) .
$$

Let us first consider the more involved derivation of equations for $\bar{x}$ and $\theta_{l}$. From equations (70), (82), and (83) we get:

$$
\frac{\mathrm{d} C_{l}}{\mathrm{~d} t}=\left(-2 i \omega\left(k_{1}\right)+2 \epsilon i \bar{x} \operatorname{Res} \Upsilon_{N N}\left(k_{1}\right)-\epsilon J_{0}\right) C_{l}-\epsilon f_{l},
$$

from which it follows that

$$
\frac{\mathrm{d}\left|C_{l}\right|^{2}}{\mathrm{~d} t}=\left[4 \operatorname{Im}\left\{\omega\left(k_{1}\right)\right\}-4 \epsilon \bar{x} \operatorname{Im}\left\{\operatorname{Res} \Upsilon_{N N}\left(k_{1}\right)\right\}-2 \epsilon \operatorname{Re}\left\{J_{0}\right\}\right]\left|C_{l}\right|^{2}-2 \epsilon \operatorname{Re}\left\{f_{l} \bar{C}_{l}\right\} .
$$

Recalling that $\sum_{l=1}^{n}\left|\theta_{l}\right|^{2}=1$ we obtain:

$$
\frac{\mathrm{d} \bar{x}}{\mathrm{~d} t}=\frac{e^{-4 \eta \bar{x}}}{4 \eta} \sum_{l=1}^{n} \frac{\mathrm{d}\left|C_{l}\right|^{2}}{\mathrm{~d} t}-\frac{\bar{x}}{\eta} \frac{\mathrm{d} \eta}{\mathrm{d} t}
$$




$$
\frac{\mathrm{d} \theta_{l}}{\mathrm{~d} t}=\theta_{l}\left(C_{l}^{-1} \frac{\mathrm{d} C_{l}}{\mathrm{~d} t}-2 \frac{\mathrm{d}(\eta \bar{x})}{\mathrm{d} t}+2 i \frac{\mathrm{d}(\xi \bar{x})}{\mathrm{d} t}\right) .
$$

The rest calculations are straightforward substitutions and using the identity $\sum_{l=1}^{n} f_{l} \bar{C}_{l}=0$, which follows from the definitions of $C_{l}, r_{0}$, and $f_{l}$. After simple calculations one gets a system of equations for the soliton parameters:

$$
\begin{gathered}
\frac{\mathrm{d} \eta}{\mathrm{d} t}=-\frac{\epsilon}{2} \int_{-\infty}^{\infty} \mathrm{d} z \operatorname{sech} z \operatorname{Re}\left\{r_{0}\right\}, \\
\frac{\mathrm{d} \xi}{\mathrm{d} t}=-\frac{\epsilon}{2} \int_{-\infty}^{\infty} \mathrm{d} z \operatorname{sech} z \tanh z \operatorname{Im}\left\{r_{0}\right\}, \\
\frac{\mathrm{d} \bar{x} \theta_{l}}{\mathrm{~d} t}=\frac{\operatorname{Im}\left\{\omega\left(k_{1}\right)\right\}}{\eta}-\frac{\epsilon}{4 \eta_{l}^{2}} \int_{-\infty}^{\infty} \mathrm{d} z z \operatorname{sech} z \operatorname{Re}\left\{r_{0}\right\}, \\
\left.\frac{2 \operatorname{Im}\left\{\bar{k}_{1} \omega\left(k_{1}\right)\right\}}{\eta}-\frac{\epsilon}{2 \eta^{2}} \int_{-\infty}^{\infty} \mathrm{d} z \operatorname{sech} z\left[\xi z \operatorname{Re}\left\{r_{0}\right\}+\eta(1-z \tanh z) \operatorname{Im}\left\{r_{0}\right\}\right]\right\} \\
+\frac{\epsilon}{4 \eta} \int_{-\infty}^{\infty} \mathrm{d} z \operatorname{sech} z\left(\theta_{l} r_{0}-e^{-i(\xi / \eta) z} r_{l}\right) .
\end{gathered}
$$

These equations can be compared with the adiabatic equations derived by Karpman [21] for the single scalar soliton. First, it is convenient to introduce the average phase $\bar{\delta}$ of the soliton by setting

$$
\bar{\delta}=\sum_{l=1}^{n}\left|\theta_{l}\right|^{2} \delta_{l}
$$

The evolution equation for the average phase then follows from equation (88):

$$
\frac{\mathrm{d} \bar{\delta}}{\mathrm{d} t}=\frac{2 \operatorname{Im}\left\{\bar{k}_{1} \omega\left(k_{1}\right)\right\}}{\eta}-\frac{\epsilon}{2 \eta^{2}} \int_{-\infty}^{\infty} \mathrm{d} z \operatorname{sech} z\left[\xi z \operatorname{Re}\left\{r_{0}\right\}+\eta(1-z \tanh z) \operatorname{Im}\left\{r_{0}\right\}\right] .
$$

Remarkably, the slow evolution of the soliton amplitude $\eta$, phase gradient $\xi$, position $\bar{x}$ and average phase $\bar{\delta}$ is given by equations similar to those derived for the single scalar soliton. The only trace of the vector nature of the soliton in equations (85)-(87) and (89) is that the "scalar" perturbation $r_{0}$ obtains by averaging the original vector perturbation as follows 


$$
r_{0}=e^{-i(\xi / \eta) z} \sum_{l=1}^{n} \bar{\theta}_{l} r_{l}
$$

The equation for $\theta_{l}=s_{l} e^{i \delta_{l}}$ can be cast in the form of two separate equations, one for the polarization parameters $s_{l}$ and the other for the phases $\delta_{l}$. We get:

$$
\begin{gathered}
\frac{\mathrm{d} s_{l}}{\mathrm{~d} t}=\frac{\epsilon}{4 \eta} \int_{-\infty}^{\infty} \mathrm{d} z \operatorname{sech} z \operatorname{Re}\left\{s_{l} r_{0}-e^{-i(\xi / \eta) z-i \delta_{l}} r_{l}\right\} \\
\frac{\mathrm{d} \delta_{l}}{\mathrm{~d} t}=\frac{2 \operatorname{Im}\left\{\bar{k}_{1} \omega\left(k_{1}\right)\right\}}{\eta}-\frac{\epsilon}{2 \eta^{2}} \int_{-\infty}^{\infty} \mathrm{d} z \operatorname{sech} z\left[\xi z \operatorname{Re}\left\{r_{0}\right\}+\eta(1-z \tanh z) \operatorname{Im}\left\{r_{0}\right\}\right] \\
+\frac{\epsilon}{4 \eta} \int_{-\infty}^{\infty} \mathrm{d} z \operatorname{sech} z \operatorname{Im}\left\{r_{0}-s_{l}^{-1} e^{-i(\xi / \eta) z-i \delta_{l}} r_{l}\right\}
\end{gathered}
$$

Note that the equation for $\delta_{l}$ contains $s_{l}$ in the denominator as a reflection of the fact that the phase is not defined for the components which are not excited.

\section{B. First-order radiation}

Now let us consider the evolution of the spectral densities $b_{l}(k)$ of radiation. Taking into account radiation in the first-order approximation amounts to solving the linearized regular Riemann-Hilbert problem (or the jump problem),

$$
\phi_{+}(k)-\phi_{-}(k)=\Gamma(k)\left(\begin{array}{cc}
0 & e^{2 i k x}|b(k)\rangle \\
\langle b(k)| e^{-2 i k x} & 0
\end{array}\right) \Gamma^{-1}(k),
$$

where we have used the notation $|b\rangle=\mathbf{b}=\left(b_{1}, \ldots, b_{n}\right)^{T}$. Solution of the above jump problem is obtained by integration and using the normalization condition $\phi_{ \pm}(k) \rightarrow I$ as $k \rightarrow \infty$. We obtain

$$
\phi(k)=I+\frac{1}{2 i \pi} \int_{-\infty}^{\infty} \frac{\mathrm{d} \ell}{\ell-k} \Gamma(\ell)\left(\begin{array}{cc}
0 & e^{2 i \ell x}|b(\ell)\rangle \\
\langle b(\ell)| e^{-2 i \ell x} & 0
\end{array}\right) \Gamma^{-1}(\ell) .
$$

The contribution from radiation to the solution follows from the formula (丑), 


$$
q_{l}^{(\mathrm{rad})}=-2 \lim _{k \rightarrow \infty} k \phi_{l N}(k)=\frac{1}{i \pi} \int_{-\infty}^{\infty} \mathrm{d} k\left\{\Gamma(k)\left(\begin{array}{cc}
0 & e^{2 i k x}|b(k)\rangle \\
\langle b(k)| e^{-2 i k x} & 0
\end{array}\right) \Gamma^{-1}(k)\right\}_{l N} .
$$

As in derivation of the equations for the soliton parameters it is convenient to use the transformation with the matrix $\Xi$. Using this transformation and formula (75) one can easily simplify the formula for radiation contribution. We get $q_{l}^{(\mathrm{rad})}=q_{l}^{(\|)}+q_{l}^{(\perp)}$, where the "parallel" and "perpendicular" parts of radiation are defined as follows

$$
\begin{gathered}
q_{l}^{(\|)}=\frac{\eta \theta_{l} e^{i(\xi / \eta) z}}{i \pi} \int_{-\infty}^{\infty} \frac{\mathrm{d} \lambda}{\lambda^{2}+1}\left\{(\lambda+i \tanh z)^{2} e^{i \lambda z}\langle\theta \mid g(\lambda)\rangle+\operatorname{sech}^{2} z e^{-i \lambda z}\langle g(\lambda) \mid \theta\rangle\right\}, \\
q_{l}^{(\perp)}=\frac{\eta e^{i(\xi / \eta) z}}{i \pi} \int_{-\infty}^{\infty} \frac{\mathrm{d} \lambda}{\lambda-i} e^{i \lambda z}(\lambda+i \tanh z)\left(g_{l}(\lambda)-\theta_{l}\langle\theta \mid g(\lambda)\rangle\right) .
\end{gathered}
$$

Here, for convenience, we have introduced the modified spectral parameter by setting $k=\eta \lambda+\xi$ and the modified spectral densities of radiation:

$$
g_{l}(\lambda)=e^{2 i(\eta \lambda+\xi) \bar{x}} b_{l}(\eta \lambda+\xi)
$$

The separation of radiation into parallel and perpendicular parts is due the following facts. While the perpendicular part $q_{l}^{(\perp)}$ satisfies the orthogonality property

$$
\sum_{l=1}^{n} \bar{\theta}_{l} q_{l}^{(\perp)}=0
$$

the parallel part of the radiation is given by the same formula as the radiation of the scalar soliton (multiplied by $\theta_{l}$ ), but for the averaged spectral density $\langle\theta \mid g(\lambda)\rangle$.

Consider the evolution equation (71) for the spectral densities of radiation. In the firstorder approximation the term with $\Upsilon_{N N}$ can be neglected. When deriving evolution equations for the modified spectral densities in the first-order approximation one must take into account only the fast (or "integrable") evolution of the soliton parameters involved in the definition of $g(\lambda)$. In particular, the modified spectral parameter $\lambda$ is $t$-independent. The only parameter which has the fast $t$-dependence in (93) is $\bar{x}$. By differentiation of (93) and using (71) and (87) we obtain 


$$
\frac{\partial g_{l}(\lambda)}{\partial t}=i \Omega_{r}(\lambda) g_{l}(\lambda)+\epsilon \theta_{l} \Upsilon^{(\|)}(\lambda)+\epsilon \Upsilon_{l}^{(\perp)}(\lambda)
$$

where

$$
\Omega_{r}(\lambda)=2\left[\lambda \operatorname{Im}\left\{\omega\left(k_{1}\right)\right\}+\operatorname{Re}\left\{\omega\left(k_{1}\right)\right\}-\omega(\eta \lambda+\xi)\right]
$$

The last two terms in equation (95) come from $\Upsilon_{l N}(79)$ (the second one is the contribution of $\left.F_{l}(\overline{77})\right)$. They read

$$
\begin{aligned}
& \Upsilon^{(\|)}(\lambda)=\frac{1}{2 \eta\left(\lambda^{2}+1\right)} \int_{-\infty}^{\infty} \mathrm{d} z e^{-i \lambda z}\left[(\lambda-i \tanh z)^{2} r_{0}-\operatorname{sech}^{2} z \bar{r}_{0}\right], \\
& \Upsilon_{l}^{(\perp)}(\lambda)=\frac{1}{2 \eta(\lambda+i)} \int_{-\infty}^{\infty} \mathrm{d} z e^{-i \lambda z}(\lambda-i \tanh z)\left(e^{-i(\xi / \eta) z} r_{l}-\theta_{l} r_{0}\right) .
\end{aligned}
$$

Due to the definition of $r_{0}(80)$, the perpendicular component satisfies the identity

$$
\sum_{l=1}^{n} \bar{\theta}_{l} \Upsilon_{l}^{(\perp)}=0
$$

Integrating the equation for $g_{l}(\lambda)$ with $g_{l}(\lambda, t=0)=0$ and using the result in (92) we arrive at the first-order correction to initially pure soliton solution. In this case we obtain:

$$
\begin{gathered}
q_{l}^{(\|)}=\epsilon \frac{\eta \theta_{l} e^{i(\xi / \eta) z}}{i \pi} \int_{-\infty}^{\infty} \frac{\mathrm{d} \lambda}{\lambda^{2}+1}\left\{(\lambda+i \tanh z)^{2} e^{i \lambda z+i \Omega_{r}(\lambda) t} \gamma^{(\|)}(\lambda)\right. \\
\left.+\operatorname{sech}^{2} z e^{-i \lambda z-i \Omega_{r}(\lambda) t} \bar{\gamma}^{(\|)}(\lambda)\right\} \\
q_{l}^{(\perp)}=\epsilon \frac{\eta e^{i(\xi / \eta) z}}{i \pi} \int_{-\infty}^{\infty} \frac{\mathrm{d} \lambda}{\lambda-i}(\lambda+i \tanh z) e^{i \lambda z+i \Omega_{r}(\lambda) t} \gamma_{l}^{(\perp)}(\lambda)
\end{gathered}
$$

where

$$
\begin{aligned}
& \gamma^{(\|)}=\frac{1}{2 \eta\left(\lambda^{2}+1\right)} \int_{-\infty}^{\infty} \mathrm{d} z e^{-i \lambda z}\left[(\lambda-i \tanh z)^{2} \hat{r}_{0}^{(+)}-\operatorname{sech}^{2} z \hat{r}_{0}^{(-)}\right] \\
& \gamma_{l}^{(\perp)}=\frac{1}{2 \eta(\lambda+i)} \int_{-\infty}^{\infty} \mathrm{d} z e^{-i \lambda z}(\lambda-i \tanh z)\left(e^{-i(\xi / \eta) z} \hat{r}_{l}-\theta_{l} \hat{r}_{0}^{(+)}\right),
\end{aligned}
$$


with

$$
\begin{gathered}
\hat{r}_{0}^{(+)}(z, t, \lambda)=\int_{0}^{t} \mathrm{~d} \tau e^{-i \Omega_{r}(\lambda) \tau} r_{0}(z, \tau), \quad \hat{r}_{0}^{(-)}(z, t, \lambda)=\int_{0}^{t} \mathrm{~d} \tau e^{-i \Omega_{r}(\lambda) \tau} \bar{r}_{0}(z, \tau), \\
\hat{r}_{l}(z, t, \lambda)=\int_{0}^{t} \mathrm{~d} \tau e^{-i \Omega_{r}(\lambda) \tau} r_{l}(z, \tau) .
\end{gathered}
$$

\section{CONCLUSIONS}

In construction of the perturbation theory our main idea is to use the Riemann-Hilbert problem associated with the integrable PDE for the nonlinear transformation of the perturbed PDE to the spectral space. The evolution equations for the spectral data follow from the evolution functional, an additional object one needs to introduce into the IST theory to account for perturbations. For a single vector soliton, the equations describing evolution of the soliton parameters and first-order radiation are given in explicit form. The method is not restricted to the first order only. For instance, the second-order equations can also be derived. The perturbation theory can be applied for description of dynamics of the spatial optical solitons, soliton pulses in the multispecies Bose-Einstein condensates, soliton propagation in optical fibre with the account of the arbitrary polarization of light pulses, and for many other applications of the multi-component soliton equations.

In this paper we have restricted the consideration to the Zakharov-Shabat spectral problem. However, the approach of this paper was successfully applied to other spectral problems as well [41-45]. There, the evolution functional was derived and the evolution equations for the spectral data were obtained. The overall result of this and the previous works on the perturbation theory based on the Riemann-Hilbert problem is that this approach always works. The explicit form of the evolution functional was the same for all considered spectral problems and, moreover, it undergoes only insignificant changes in the transition from the Cauchy problem to an initial-boundary value problem [45]. 


\section{ACKNOWLEDGMENTS}

The author is indebted to Professor E. V. Doktorov for stimulating discussions during the course of this work and his critical reading of the manuscript. This research was supported in part by the NRF of South Africa.

\section{APPENDIX A: SOME COMMENTS ON MULTIPLICITY OF ZEROS}

Here we explore in more detail the algebraic and geometric multiplicities of zeros of $\operatorname{det} \Phi_{+}(k)$ and $\operatorname{det} \Phi_{-}^{-1}(k)$. For instance, consider the determinant

$$
\operatorname{det} \Phi_{+}=J_{+1} \wedge \ldots \wedge J_{+n} \wedge J_{-n+1} \wedge \ldots \wedge J_{-N}
$$

None of the columns $J_{ \pm_{l}}(x, k)$ is equal to zero (otherwise, due to uniqueness of solution to equation (11) we would have $J_{ \pm l}=0$ for all $x$ ). Moreover, the minors in the wedge products of columns of $J_{+}$and $J_{-}$satisfy linear homogeneous equations, which follow from (1), section III,

$$
\begin{aligned}
\partial_{x} J_{+1} \wedge \ldots \wedge J_{+n}=\left\{i k\left(\hat{A}^{(n)}-\frac{n(n+1)}{2} \hat{I}^{(n)}\right)+i \hat{Q}^{(n)}\right\} J_{+1} \wedge \ldots \wedge J_{+n}, \\
\partial_{x} J_{-n+1} \wedge \ldots \wedge J_{-N}=\left\{i k\left(\hat{A}^{(N-n)}-\frac{(N-n)(N-n+1)}{2} \hat{I}^{(N-n)}\right)+i \hat{Q}^{(N-n)}\right\} \\
\times J_{-n+1} \wedge \ldots \wedge J_{-N} .
\end{aligned}
$$

Here $\hat{M}^{(j)}$ denotes a super-matrix, whose action on the wedge products of vector-columns is defined by the rule

$$
\hat{M}^{(j)} \Psi_{1} \wedge \ldots \wedge \Psi_{j}=\sum_{p=1}^{j} \Psi_{1} \wedge \ldots \wedge M \Psi_{p} \wedge \ldots \wedge \Psi_{j}
$$

Therefore, $\operatorname{rank} J_{+1} \wedge \ldots \wedge J_{+n}=\operatorname{rank} e_{1} \wedge \ldots \wedge e_{n}=n$, and $\operatorname{rank} J_{-n+1} \wedge \ldots \wedge J_{-N}=$ $\operatorname{rank} e_{n+1} \wedge \ldots \wedge e_{N-n}=N-n$. Hence, the only possibility for $\operatorname{det} \Phi_{+}(k)=0$ is the linear 
dependence of the columns in $J_{+} H_{1}$ and $J_{-} H_{2}$, e.g., at least one of the columns of $J_{-} H_{2}$ is given as a linear combination of the columns of $J_{+} H_{1}$.

Let $\nu_{j}$ and $d_{j}$ be the algebraic and geometric multiplicities of the $j$-th zero $k_{j}$, i.e.,

$$
\operatorname{det} \Phi_{+}(k)=\left(k-k_{j}\right)^{\nu_{j}} \psi(k), \quad \psi\left(k_{j}\right) \neq 0, \quad d_{j}=N-\operatorname{rank} \Phi_{+}\left(k_{j}\right) .
$$

Writing down the Taylor expansion of $\Phi_{+}(k)$ about $k=k_{j}$ we immediately conclude that

$$
\operatorname{rank} \Phi_{+}\left(k_{j}\right) \geq N-\nu_{j}
$$

Therefore, in general, the algebraic multiplicity is greater than the geometric one; trivially, they coincide for simple zeros. Representation (A1) gives

$$
\operatorname{rank} \Phi_{+}\left(k_{j}\right) \geq \max (n, N-n)
$$

Hence the geometric multiplicity satisfies

$$
d_{j} \leq N-\max (n, N-n)
$$

We consider only zeros whose algebraic multiplicity is equal to the geometric one. In particular, if $n=N-1$, there can be only one vector in the null space of $\Phi_{+}$, i.e., $d_{j}=1$. Hence, in this case, zeros of $\operatorname{det} \Phi_{+}(k)$ must be simple to satisfy the equal multiplicity condition. This condition requires that the only case of a multiple zero, $\operatorname{det} \Phi_{+}\left(k_{j}\right)=0$, of order $\nu_{j}$ is that there are precisely $\nu_{j}$ columns of $J_{-}\left(k_{j}\right) H_{2}$ and $J_{+}(x, k) H_{1}$ which are linear combinations of the columns of $J_{+}\left(k_{j}\right) H_{1}$ and $J_{-}\left(k_{j}\right) H_{2}$, respectively.

The equal multiplicity condition can be guaranteed by the following constraint (imposed for some $(x, t))$

$$
\operatorname{rank}\left(\Phi_{+}, \frac{\partial \Phi_{+}}{\partial k}\right)=N
$$

Indeed, for algebraic multiplicity $\nu_{j}$, by the Taylor expansion, (A4) gives $\operatorname{rank} \Phi_{+}\left(k_{j}\right)=$ $N-\nu_{j}$. Conversely, if $\operatorname{rank} \Phi_{+}\left(k_{j}\right)=N-\nu_{j}$, for algebraic multiplicity $\nu_{j}$, then there are at least $\nu_{j}$ columns in $\partial \Phi_{+}\left(k_{j}\right) / \partial k$ independent from the columns of $\Phi_{+}\left(k_{j}\right)$. Hence (A4) also holds. Similar results are valid for multiplicities of zeros $\bar{k}_{j}, j=1, \ldots, s$, of $\operatorname{det} \Phi_{-}^{-1}(k)$. 


\section{APPENDIX B: PROPERTIES OF THE REGULARIZATION MATRIX}

Here we derive the regularization matrix $\Gamma(k)$ and prove its properties (in the main, we follow Refs. 13 and 58). Dependence on the co-ordinates $x$ and $t$ is not important for this purpose and omitted. Consider one pair of zeros, say, $k_{s}$ and $\bar{k}_{s}$ of $\operatorname{det} \Phi_{+}(k)$ and $\operatorname{det} \Phi_{-}^{-1}(k)$, respectively. Let the vectors $\left|p_{l}^{(s)}\right\rangle$ and $\left\langle\bar{p}_{l}^{(s)}\right|, l=1, \ldots, \nu_{s}$, satisfying

$$
\Phi_{+}\left(k_{s}\right)\left|p_{l}^{(s)}\right\rangle=0, \quad\left\langle\bar{p}_{l}^{(s)}\right| \Phi_{-}^{-1}\left(\bar{k}_{s}\right)=0
$$

span the respective null spaces. Construct the following rational matrix functions

$$
\chi_{s}(k)=I-\frac{k_{s}-\bar{k}_{s}}{k-\bar{k}_{s}} P_{s}, \quad \bar{\chi}_{s}(k)=I+\frac{k_{s}-\bar{k}_{s}}{k-k_{s}} P_{s}
$$

where

$$
P_{s}=\sum_{l, m=1}^{\nu_{s}}\left|p_{l}^{(s)}\right\rangle\left(M^{-1}\right)_{l m}\left\langle\bar{p}_{m}^{(s)}\right|, \quad M_{l m}=\left\langle\bar{p}_{l}^{(s)} \mid p_{m}^{(s)}\right\rangle
$$

and $P_{s}$ is a projector: $P_{s}^{2}=P_{s}, \operatorname{rank} P_{s}=\nu_{s}$. It is easy to verify that $\bar{\chi}_{s}(k)$ is inverse to $\chi_{s}(k): \chi_{s}(k) \bar{\chi}_{s}(k)=I$.

The determinant of $\chi_{s}(k)$ is easily computed in some appropriate basis, where the projector is represented by a diagonal matrix with $\nu_{s}$ ones and $N-\nu_{s}$ zeros on the diagonal. We get

$$
\operatorname{det} \chi_{s}(k)=\left(\frac{k-k_{s}}{k-\bar{k}_{s}}\right)^{\nu_{s}}
$$

Hence, with such rational matrices we can factor out the $s$-th pair of zeros. Indeed, consider the products $\Phi_{+}(k) \chi_{s}^{-1}(k)$ and $\chi_{s}(k) \Phi_{-}^{-1}(k)$. These matrix functions are holomorphic in the upper and lower half planes, respectively (the poles are removable due to the identities (B1)). On the other hand, the determinants are non-zero for $k=k_{s}$ and $k=\bar{k}_{s}$, respectively; thus one pair of zeros is factored out. By introducing a sequence of such matrices,

$$
\chi_{j}(k)=I-\frac{k_{j}-\bar{k}_{j}}{k-\bar{k}_{j}} P_{j}, \quad \bar{\chi}_{j}(k)=I+\frac{k_{j}-\bar{k}_{j}}{k-k_{j}} P_{j}, \quad j=1, \ldots, s,
$$


where $\bar{\chi}_{j}(k)=\chi_{j}^{-1}(k)$, we factor out all zeros using the regularization matrix $\Gamma$ and its inverse, where

$$
\Gamma(k)=\chi_{1}(k) \chi_{2}(k) \cdot \ldots \cdot \chi_{s}(k) .
$$

The projector $P_{j}$ is given by the following formula

$$
P_{j}=\sum_{l, m=1}^{s}\left|e_{l}^{(j)}\right\rangle\left(M^{-1}\right)_{l m}\left\langle\bar{e}_{m}^{(j)}\right|, \quad M_{l m}=\left\langle\bar{e}_{l}^{(j)} \mid e_{m}^{(j)}\right\rangle .
$$

Here the vectors $\left|e_{l}^{(j)}\right\rangle$ and $\left\langle\bar{e}_{l}^{(j)}\right|$ are related to the basis vectors of the null spaces of $\Phi_{+}\left(k_{j}\right)$ and $\Phi_{-}^{-1}\left(\bar{k}_{j}\right)$ by triangular equations (if scanned starting from $s$ down to 1 ):

$$
\begin{gathered}
\left|p_{l}^{(j)}\right\rangle=\chi_{s}^{-1}\left(k_{j}\right) \cdot \chi_{s-1}^{-1}\left(k_{j}\right) \cdot \ldots \cdot \chi_{j+1}^{-1}\left(k_{j}\right)\left|e_{l}^{(j)}\right\rangle \\
\left\langle\bar{e}_{l}^{(j)}\right| \chi_{j+1}\left(\bar{k}_{j}\right) \cdot \chi_{j+2}\left(\bar{k}_{j}\right) \cdot \ldots \cdot \chi_{s}\left(\bar{k}_{j}\right)=\left\langle\bar{p}_{l}^{(j)}\right| .
\end{gathered}
$$

Due to $P_{j}^{2}=P_{j}$, these vectors satisfy the identities:

$$
\chi_{j}\left(k_{j}\right)\left|e_{l}^{(j)}\right\rangle=0, \quad\left\langle\bar{e}_{l}^{(j)}\right| \chi_{j}^{-1}\left(k_{j}\right)=0, \quad l=1, \ldots, \nu_{j}
$$

The regularization matrix $\Gamma(k)$ can be made parameterized entirely by the vectors from the null spaces. Indeed, let us decompose $\Gamma(k)$ and the inverse matrix into the partial fractions:

$$
\Gamma(k)=I-\sum_{j=1}^{s} \frac{\bar{B}_{j}}{k-\bar{k}_{j}}, \quad \Gamma^{-1}(k)=I+\sum_{j=1}^{s} \frac{B_{j}}{k-k_{j}},
$$

where due to $(\mathrm{B} 4)$ and $(\mathrm{B} 5)$ we have

$$
B_{j}=\sum_{l=1}^{\nu_{j}}\left|p_{l}^{(j)}\right\rangle\left\langle v_{l}^{(j)}\left|, \quad \bar{B}_{j}=\sum_{l=1}^{\nu_{j}}\right| \bar{v}_{l}^{(j)}\right\rangle\left\langle\bar{p}_{l}^{(j)}\right| .
$$

From (B7) and the identity $\Gamma \Gamma^{-1}=\Gamma^{-1} \Gamma=I$ it follows that

$$
\Gamma\left(k_{j}\right)\left|p_{l}^{(j)}\right\rangle=0, \quad\left\langle\bar{p}_{l}^{(j)}\right| \Gamma^{-1}\left(\bar{k}_{j}\right)=0, \quad l=1, \ldots, \nu_{j}, \quad j=1, \ldots, s .
$$

These are the equations defining the unknown vectors $\left|\bar{v}_{l}^{(j)}\right\rangle$ and $\left\langle v_{l}^{(j)}\right|$. Indeed, rewriting (B9) we have 


$$
\left|p_{l}^{(j)}\right\rangle=\sum_{i=1}^{s} \frac{1}{k_{j}-\bar{k}_{i}} \sum_{m=1}^{\nu_{i}}\left|\bar{v}_{m}^{(i)}\right\rangle\left\langle\bar{p}_{m}^{(i)} \mid p_{l}^{(j)}\right\rangle, \quad\left\langle\bar{p}_{l}^{(j)}\right|=-\sum_{i=1}^{s} \frac{1}{\bar{k}_{j}-k_{i}} \sum_{m=1}^{\nu_{i}}\left\langle\bar{p}_{l}^{(j)} \mid p_{m}^{(i)}\right\rangle\left\langle v_{m}^{(i)}\right| .
$$

Inversion of these formulae gives

$$
\left|\bar{v}_{l}^{(j)}\right\rangle=\sum_{i=1}^{s} \sum_{m=1}^{\nu_{i}}\left|p_{m}^{(i)}\right\rangle\left(D^{-1}\right)_{i m, j l}, \quad\left\langle v_{l}^{(j)}\right|=\sum_{i=1}^{s} \sum_{m=1}^{\nu_{i}}\left(D^{-1}\right)_{j l, i m}\left\langle\bar{p}_{m}^{(i)}\right| .
$$

Here the matrix $D$ is defined by

$$
D_{i m, j l}=\frac{\left\langle\bar{p}_{m}^{(i)} \mid p_{l}^{(j)}\right\rangle}{k_{j}-\bar{k}_{i}} .
$$

Substitution of (B10) into (B7) produces the needed formulae (29). 


\section{REFERENCES}

[1] A. V. Mikhailov et al, Russ. Math. Surveys 42, 1 (1987); J. Hietarinta, Phys. Rep. 147, 87 (1987).

[2] M. Mitchell, Z. Chen, M. Shin, and M. Segev, Phys. Rev. Lett. 77, 490 (1996); M. Mitchel and M. Segev, Nature (London) 387, 880 (1997).

[3] M. Mitchell, M. Segev, T. H. Coskun, and D. N. Christodoulides, Phys. Rev. Lett. 79, 4990 (1997); D. N. Christodoulides, T. H. Coskun, M. Mitchell and M. Segev, Phys. Rev. Lett. 80, 2310 (1998); D. N. Christodoulides, T. H. Coskun, M. Mitchell, Z. Chen, and M. Segev Phys. Rev. Lett. 80, 5113 (1998); N. N. Akhmediev, W. Królikowski, and A. W. Snyder, Phys. Rev. Lett. 81, 4632 (1998); A. A. Sukhorukov and N. N. Akhmediev, Phys. Rev. Lett. 83, 4736 (1999); O. Bang, D. Edmindon, and W. Królikowski, Phys. Rev. Lett. 83, 5479 (1999).

[4] C. K. Law, H. Pu, N. B. Bigelow, and J. H. Eberly, Phys. Rev Lett. 79, 3105 (1997); T.-L. Ho, Phys. Rev. Lett. 81, 742 (1998); S.-K. Yip, Phys. Rev. Lett. 83, 4677 (1999).

[5] G. P. Agrawal, Nonlinear Fiber Optics (Academic Press, New York, 1989); Optical Solitons - Theory and Experiment ed. J. R. Taylor, Cambridge Studies in Modern Optics 10 (Cambridge University Press, Cambridge, England 1992).

[6] S. Chakravarty, M. J. Ablowitz, J. R. Sauer and R. B. Jenkins, Opt. Lett. 20, 136 (1995).

[7] C. Yen and L. A. Bergman, Phys Rev E 57, 2398 (1998); 60, 2306 (1999).

[8] F. T. Hioe, Phys. Rev. Lett. 82, 1152 (1999).

[9] B. Tan and J. P. Boyd, Chaos, Solitons \& Fractals 11, 1113 (2000).

[10] A. V. Mikhailov, Physica D 3, 73 (1981); A. P. Fordy and P. P. Kulish, Commun. Math. Phys. 89, 427 (1983).

[11] V. E. Zakharov and A. B. Shabat Sov. Phys. JETP 34, 62 (1972); Funct. Anal. Appl. 
8, 226 (1974); 13, 13 (1979).

[12] S. V. Manakov, Zh. Eksp. Teor. Fiz. 65, 505 (1973) [Sov. Phys. JETP 38, 248 (1974)].

[13] S. P. Novikov, S. V. Manakov, L. P. Pitaevski, and V. E. Zakharov, Theory of Solitons the Inverse Scattering Method (Consultants Bureau, New York, 1984).

[14] K. Nakkeeran, K. Porsezian, S. P. Shanmugha, and A. Mahalingam, Phys. Rev. E 80, 1425 (1998).

[15] T. Tsuchida and M. Wadati, J. Phys. Soc. Jpn. 67, 1175 (1998); Phys. Lett. A 257, 53 (1999); Inverse Problems 15, 1363 (1999).

[16] S. Yu. Sakovich and T. Tsuchida, J. Phys. A: Math. Gen. 33, 7217 (2000).

[17] F. M. Mitschke and L. F. Mollenauer, Opt. Lett. 11, 657 (1986); Y. Kodama and A. Hasegawa, IEEE J. Quantum Electron. 23, 510 (1987).

[18] A. Hasegawa, Optical Solitons in Fibers (Springer, Heidelberg, 1989).

[19] A. Hasegawa and Y. Kodama, Solitons in Optical Communications (Clarendon Press, Oxford, 1995).

[20] D. J. Kaup, SIAM J. Appl. Math. 31, 121 (1976); D. J. Kaup and A. C. Newell, Proc. R. Soc. Lond. Ser. A 361, 413 (1978).

[21] V. I. Karpman, Phys. Scr. 20, 462 (1979); V. I. Karpman and E. M. Maslov, Sov. Phys. JETP 46, 281 (1977).

[22] J. Kaup, J. Math. Anal. Appl. 54, 849 (1976); J. Math. Phys. 25, 2467 (1984).

[23] V. S. Gerdjikov, M. I. Ivanov, and P. P. Kulish, Teor. Mat. Fiz. 44, 342 (1980) (in Russian).

[24] T. Kawata, J. Phys. Soc. Jpn. 53, 2879 (1984).

[25] K. Iino and Y. Ichikawa, J. Phys. Soc. Jpn. 51, 4091 (1982); 51, 2389 (1982). 
[26] V. S. Gerdjikov and A. B. Yanovski, Phys. Lett. A. 103, 232 (1984); Phys. Lett. A 110, 53 (1985); Commun. Math. Phys. 103, 545 (1986).

[27] V. S. Gerdjikov, Theor. Math. Phys. 92, 374 (1992); V. S. Gerdjikov, M. I. Ivanov, Inverse Problems 8, 831 (1992).

[28] D. J. Kaup and T. I. Lakoba, J. Math. Phys. 37, 308 (1996); T. I. Lakoba and D. J. Kaup, Phys. Rev. E 56, 6147 (1997).

[29] J. P. Keener and D. W. Mclaughlin, Phys. Rev. A 16, 777 (1977).

[30] Yu. S. Kivshar, Physica D 40, 11 (1989).

[31] Yu. S. Kivshar and B. A. Malomed, Rev. Mod. Phys. 61, 763 (1989).

[32] D. J. Kaup, Phys. Rev. A 42, 5689 (1990).

[33] R. L. Herman, J. Phys. A: Math. Gen. 23, 2327 (1990).

[34] E. V. Doktorov and I. N. Prokopenya, Inv. Probl. 7, 221 (1991); Zh. Eksp. Teor. Fiz. 100, 1129 (1991) [Sov. Phys. JETP 73, 623 (1991)].

[35] V. V. Konotop and V. E. Vekslerchik, Phys. Rev. E 49, 2397

[36] D. E. Pelinovsky, A. V. Buryak, and Yu. S. Kivshar, Phys. Rev. Lett. 75, 591 (1995);

D. E. Pelinovsky, Yu. S. Kivshar, and V. V. Afanasjev, Phys. Rev. E 53, 1940 (1996).

[37] I. V. Barashenkov, D. E. Pelinovsky, and E. V. Zemlyanaya, Phys. Rev. Lett. 80, 5117 (1998).

[38] N. V. Alexeeva, I. V. Barashenkov, and D. E. Pelinovsky, Nonlinearity 12, 103 (1999).

[39] J. Yang and D. J. Kaup, SIAM J. Appl. Math. 60, 967 (2000).

[40] J. Yang, J. Math. Phys. 41, 6614 (2000).

[41] V. S. Shchesnovich, Chaos, Solitons \& Fractals, 5, 2121 (1995). 
[42] V. S. Shchesnovich and E. V. Doktorov, Phys. Rev. E 55, 7626 (1997).

[43] V. S. Shchesnovich and E. V. Doktorov, Physica D 129, 115 (1999).

[44] V. S. Shchesnovich and I. V. Barashenkov, in preparation.

[45] E. V. Doktorov and V. S. Shchesnovich, Inverse Problems 17, 971 (2001).

[46] M. J. Ablowitz and P. A. Clarkson, Solitons, Nonlinear Evolution Equations and Inverse Scattering (Cambridge University Press, Cambridge, 1991).

[47] A. S. Fokas, SIAM J. Math. Anal. 27, 738 (1996); Proc. R. Soc. Lond. A 453, 1411 (1997); J. Math. Phys. 41, 4188 (2000).

[48] L. D. Faddeev and L. A. Takhtajan, Hamiltonian Methods in the Theory of Solitons (Springer-Verlag, Berlin-Heidelberg-New York, 1987).

[49] J. Leon, J. Math. Phys. 35, 3504 (1994).

[50] A. C. Newell, Solitons in Mathematics and Physics (SIAM, Philadelphia 1985).

[51] M. J. Ablowitz and H. Segur, Solitons and the Inverse Scattering Transform (SIAM, Philadelphia 1981).

[52] F. Calogero and A. Degasperis, Spectral Transform and Solitons (North-Holland, Amsterdam, 1982).

[53] V. S. Gerdjikov, M. I. Ivanov, P. P. Kulish, Lett. Math. Phys. 6, 315 (1982).

[54] X. Zhou, SIAM J. Math. Anal. 20, 966 (1988); Commun. Pure Appl. Math. 42, 895 (1989).

[55] R. Beals and R. R. Coifman, Commun. Pure Appl. Math. 37, 39 (1984); 38, 29 (1985); Inverse Problems 5577 (1989).

[56] R. Beals, P. Deift, and C. Tomei, Direct and Inverse Scattering on the Line, Math. Surv. Mono., 28, American Mathematical Society, Providence, R. I., 1988. 
[57] P. J. Caudrey, Physica D 6, 51 (1982).

[58] T. Kawata, Riemann spectral method for the nonlinear evolution equations, p. 210, in Advances in Nonlinear Waves, ed. by L. Debnath, (Cambridge Univ. Press, Cambridge, 1984). 